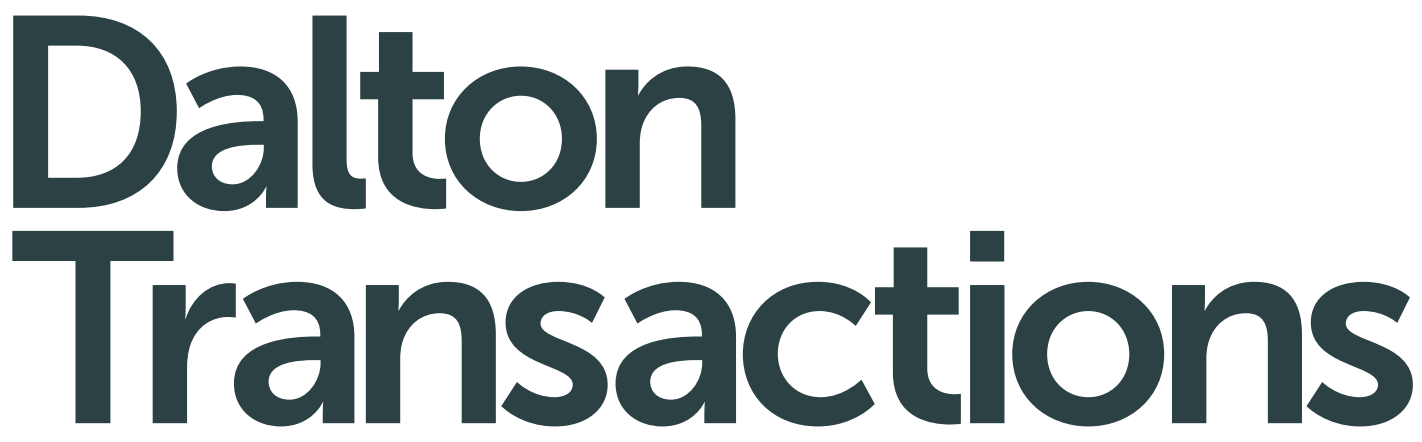

An international journal of inorganic chemistry www.rsc.org/dalton

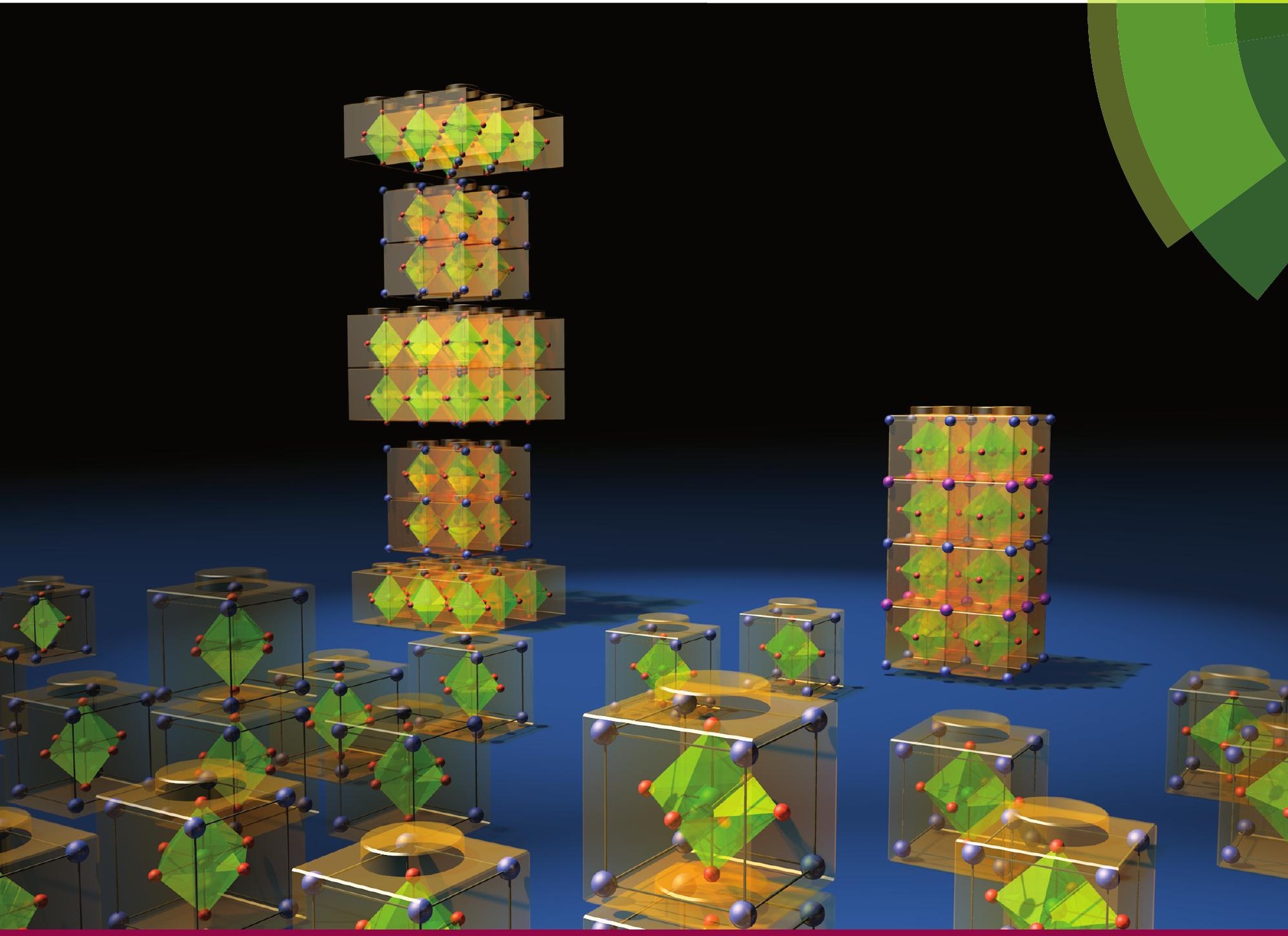

Themed issue: Perovskites

ISSN $1477-9226$

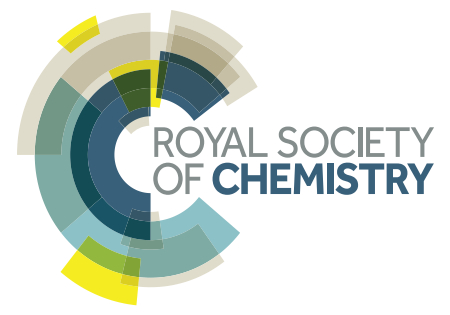




\title{
Understanding ferroelectricity in layered perovskites: new ideas and insights from theory and experiments
}

\author{
Nicole A. Benedek, ${ }^{* a}$ James M. Rondinelli, ${ }^{b}$ Hania Djani, ${ }^{c}$ Philippe Ghosez ${ }^{d}$ and \\ Philip Lightfoot ${ }^{\mathrm{e}}$
}

\begin{abstract}
$\mathrm{ABO}_{3}$ perovskites have fascinated solid-state chemists and physicists for decades because they display a seemingly inexhaustible variety of chemical and physical properties. However, despite the diversity of properties found among perovskites, very few of these materials are ferroelectric, or even polar, in bulk. In this Perspective, we highlight recent theoretical and experimental studies that have shown how a combination of non-polar structural distortions, commonly tilts or rotations of the $\mathrm{BO}_{6}$ octahedra, can give rise to polar structures or ferroelectricity in several families of layered perovskites. We discuss the crystal chemical origin of the polarization in each of these families - which emerges through a so-called 'trilinear coupling' or 'hybrid improper' mechanism - and emphasize areas in which further theoretical and experimental investigation is needed. We also consider how this mechanism may provide a generic route for designing not only new ferroelectrics, but also materials with various other multifunctionalities, such as magnetoelectrics and electric field-controllable metal-insulator transitions.
\end{abstract}

Received 3rd January 2015, Accepted 6th February 2015

DOI: $10.1039 / \mathrm{c} 5 \mathrm{dt} 00010 \mathrm{f}$

www.rsc.org/dalton ferroics. ${ }^{4,5}$ The origin of ferroelectricity in both of these materials is a pseudo- ${ }^{6,7}$ or Second-Order Jahn-Teller distortion (SOJT). ${ }^{8}$ In the case of $\mathrm{BaTiO}_{3}$, the softness of the polar distortion is driven by hybridization between the formally empty $\mathrm{Ti} 3 \mathrm{~d}$ states and formally filled $\mathrm{O} 2 \mathrm{p}$ states; ${ }^{9,10}$ the stereochemically active lone pair on $\mathrm{Bi}^{3+}$ is responsible for ferroelectricity in $\mathrm{BiFeO}_{3}{ }^{11,12}$ However, although $\mathrm{BaTiO}_{3}$ and $\mathrm{BiFeO}_{3}$ are commonly thought of as typical perovskite ferroelectrics, the vast majority of $\mathrm{ABO}_{3}$ perovskites are not ferroelectric, or even polar. ${ }^{13}$

In contrast to bulk $\mathrm{ABO}_{3}$ perovskites, the family of layered perovskite-like phases contains a number of polar and ferroelectric materials. For example, the Aurivillius phase $^{14}$ $\mathrm{SrBi}_{2} \mathrm{Ta}_{2} \mathrm{O}_{9}$ has been extensively investigated ${ }^{15-17}$ as a fatiguefree ferroelectric for ferroelectric memories. ${ }^{18,19} \mathrm{Bi}_{4} \mathrm{Ti}_{3} \mathrm{O}_{12}$, $\mathrm{Bi}_{3} \mathrm{TiNbO}_{9}$ and $\mathrm{Bi}_{2} \mathrm{WO}_{6}{ }^{20,21}$ are also Aurivillius ferroelectrics. ${ }^{22}$ The Ruddlesden-Popper phases ${ }^{23,24} \quad \mathrm{Ca}_{3} \mathrm{Ti}_{2} \mathrm{O}_{7},{ }^{25}$ $\mathrm{Ca}_{3} \mathrm{Mn}_{2} \mathrm{O}_{7}{ }^{26,27}$ and $\mathrm{Ca}_{3} \mathrm{Ru}_{2} \mathrm{O}_{7}{ }^{28}$ are all polar, although the parent materials $\left(\mathrm{CaTiO}_{3}, \mathrm{CaMnO}_{3}\right.$ and $\left.\mathrm{CaRuO}_{3}\right)$ are not. The Dion-Jacobson phases ${ }^{29,30} \mathrm{CsBiNb}_{2} \mathrm{O}_{7}$ and $\mathrm{CsNdNb}_{2} \mathrm{O}_{7}$ have been experimentally observed ${ }^{31,32}$ to be polar and ferroelectricity has been demonstrated in $\mathrm{RbBiNb}_{2} \mathrm{O}_{7}{ }^{33}$ and, very recently, in $\mathrm{CsBiNb}_{2} \mathrm{O}_{7} \cdot{ }^{34}$ First-principles calculations have predicted that several other Dion-Jacobson phases may also be polar. ${ }^{35} \mathrm{~A}$ number of $\mathrm{AA}^{\prime} \mathrm{B}_{2} \mathrm{O}_{6}$ double perovskites ${ }^{36,37}$ are either known experimentally to be polar (for example, $\mathrm{NaLaMnWO}_{6}{ }^{38}{ }^{3}$ which exhibits simultaneous A- and B-site cation ordering) or have 

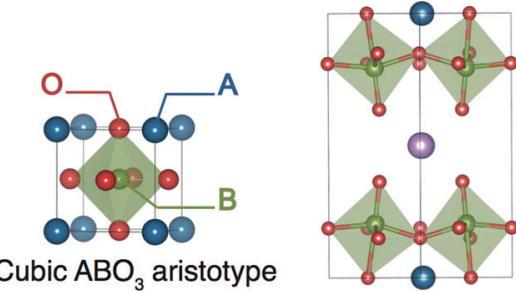

$A^{\prime}\left[A_{n-1} B_{n} O_{3 n+1}\right]$

Dion-Jacobson

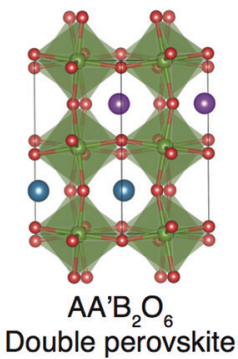

Double perovskite

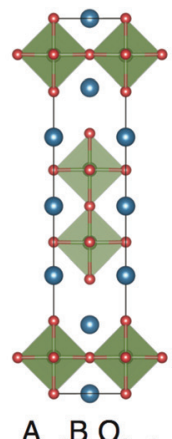

$\mathrm{A}_{\mathrm{n}+1} \mathrm{~B}_{\mathrm{n}} \mathrm{O}_{3 \mathrm{n}+1}$

RuddlesdenPopper

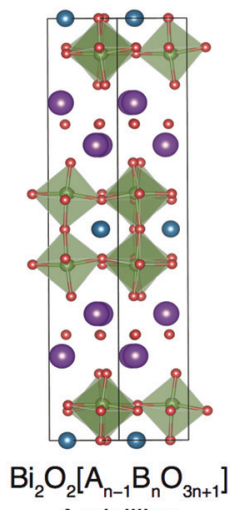

Aurivillius

Fig. 1 Layered perovskites - derived from the cubic $\mathrm{ABO}_{3}$ aristotype - discussed in this Perspective. In the Dion-Jacobson phases, $A^{\prime}$ is usually an alkali cation, but can also be a transition metal halide complex, e.g. $(\mathrm{MnCl})^{+}$. In the A-site ordered double perovskites, $\mathrm{A}^{\prime}$ is chemically different to A. The perovskite blocks are interleaved between $\left[\mathrm{Bi}_{2} \mathrm{O}_{2}\right]^{2+}$ layers in the Aurivillius phases.

been predicted to be polar from first-principles calculations. $^{39-41}$ Ferroelectricity has also been demonstrated in various artificial superlattices grown as thin-films, ${ }^{42-46}$ even in cases it was unexpected. ${ }^{47}$ Why is ferroelectricity - and acentricity in general - much more common among the layered perovskites than their $\mathrm{ABO}_{3}$ counterparts?

In this Perspective, we review recent progress in understanding the crystal chemistry of layered perovskites, ${ }^{48}$ particularly the mechanisms by which polar structures emerge in various families of materials; see Fig. 1. In contrast to SOJTdriven materials like $\mathrm{BaTiO}_{3}$ and $\mathrm{BiFeO}_{3}$, the origin of the transition from a non-polar to a polar structure in many layered perovskites is not the instability of a polar mode driven by charge transfer or hybridization. Instead, the transition involves two non-polar lattice distortions (commonly 'rotations' or 'tilts' of the $\mathrm{BO}_{6}$ octahedra), which couple to a polar lattice mode in a so-called 'trilinear coupling,49 or 'hybrid improper' ${ }^{50}$ mechanism. The importance of octahedral rotations (which are generally driven by electrostatic or ion size mismatch effects) in giving rise to polar structures was emphasized in several experimental studies of Aurivillius phases, Dion-Jacobson phases and double perovskites. ${ }^{22,31,32,36-38}$ These works essentially anticipated the trilinear coupling mechanism as the origin of ferroelectricity, the microscopics of which was first revealed by Bousquet et al. ${ }^{47}$ and further formally elucidated in a series of papers using theory and firstprinciples calculations. ${ }^{39,40,49-53}$ We begin by briefly reviewing the basics of this mechanism from the perspective of theory, and discuss how it manifests in different families of layered perovskites. In particular, we aim to highlight advances in understanding made possible by synergistic interactions between theory and experiment. Finally, we discuss the possibilities for exploiting the trilinear coupling mechanism as a generic route to creating various types of multifunctionalities,

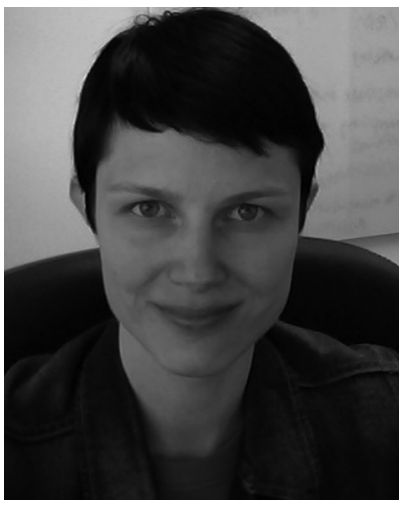

Nicole A. Benedek
Nicole A. Benedek is an Assistant Professor in the Materials Science and Engineering Program at The University of Texas at Austin, where she leads the Theory and Simulation of Materials Group. She holds a PhD in Applied Physics and Chemistry from RMIT University in Melbourne, Australia. Nicole is the recipient of a Ralph E. Powe Junior Faculty Enhancement Award (2014) and a NSF-CAREER award (2015). The Benedek group uses first-principles theoretical techniques to explore and solve fundamental problems in the solid-state chemistry of complex oxides, with a particular interest in polar materials, ionic and thermal transport.

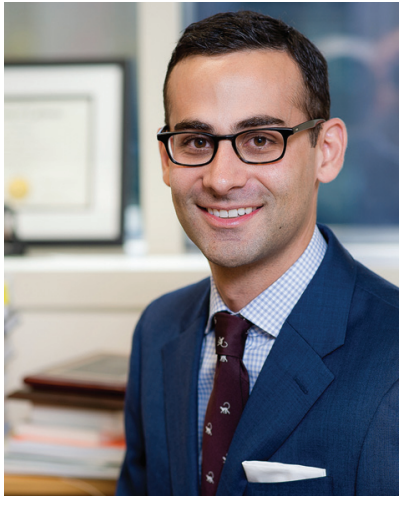

James Rondinelli
Dr James Rondinelli is an assistant professor at Northwestern University in the Materials Science and Engineering (MSE) Department, where he leads the Materials Theory and Design Group. He received his $P h D$ in materials science from the University of California, Santa Barbara in 2010. Some of his honors include receiving the NSF-CAREER Award (2015), DARPA Young Faculty Award (2012), and ARO Young Investigator Program (YIP) award (2012). At Northwestern, his interests are in the first-principles design and understanding of functional complex oxide- and fluoride-based materials-inorganic compounds that are expected to be key in next generation electronic and optical devices. 
such as metal-insulator transitions and magnetoelectric effects.

\section{Trilinear coupling: polar structures from non-polar lattice distortions}

Before discussing the specifics of the trilinear coupling mechanism, it is useful to briefly review the manner in which polar structures and ferroelectricity emerge in prototypical ferroelectrics like $\mathrm{BaTiO}_{3}$ and $\mathrm{PbTiO}_{3}$. In these materials, ferroelectricity is driven by a single, zone-center polar lattice distortion (phonon), which gives rise to a spontaneous polarization. The cooperative acentric displacements associated with this polar lattice mode (sketched in Fig. 2a), completely account for the symmetry lost between the paraelectric parent phase and the ferroelectric ground state. ${ }^{54}$ A convenient approach to understand the loss of inversion symmetry at the atomic scale involves treating the electric polarization that results from the displacements as an order parameter, and expanding the free energy $\mathcal{F}$ of the paraelectric phase in powers of the polarization $P$ (to fourth order) as:

$$
\mathcal{F}=\mathcal{F}_{0}+\alpha P^{2}+\beta P^{4},
$$

where $\mathcal{F}_{0}$ is the energy of the undistorted paraelectric phase and $\alpha$ and $\beta$ are coefficients. Ferroelectric transitions for which the polarization is the primary order parameter are known as 'proper'. Below the Curie temperature $(\alpha<0)$, eqn (1) produces the double-well potential energy curve that is shown in Fig. 2c, and is characteristic of proper ferroelectrics.

The polarization is not the primary order parameter in all ferroelectric transitions, as in the case of 'improper' ferroelectrics ${ }^{55}$ such as the hexagonal multiferroic $\mathrm{YMnO}_{3} \cdot{ }^{56,57}$ The polarization is instead coupled to another primary cooperative atomic displacement pattern $R$, which occurs at the zone- boundary of the paraelectric phase and thus leads to an enlargement of the unit cell at the transition. For example, Fig. $2 b$ depicts such a mode in the 5 -atom cubic perovskite cell, which manifest as rotations of the $\mathrm{BO}_{6}$ octahedra. In hexagonal $\mathrm{YMnO}_{3}$, a similar type of zone-boundary mode, which triples the unit cell of the paraelectric phase and leads to a tilting of the $\mathrm{MnO}_{5}$ polyhedra and a buckling of the $\mathrm{Y}-\mathrm{O}$ planes, acts as the primary order parameter driving the transition into the ferroelectric ground state. In other words, the polar zonecenter mode is not intrinsically unstable in improper ferroelectrics and only appears due to its coupling with the zoneboundary mode (Fig. 2e).

In the specific case of $\mathrm{YMnO}_{3}$, (or rather, any isostructural hexagonal manganite and even $\mathrm{BaMnO}_{3},{ }^{58}$ in spite of its very different crystal structure), the free energy expansion in terms of $P$ and $R$ can be written as, ${ }^{57,59}$

$$
\begin{aligned}
\mathcal{F}= & \mathcal{F}_{0}+\alpha_{20} R^{2}+\alpha_{02} P^{2}+\beta_{40} R^{4} \\
& +\beta_{04} P^{4}+\beta_{31} R^{3} P+\beta_{22} R^{2} P^{2} .
\end{aligned}
$$

The key detail is the second-last term, which couples the polarization at linear order to the primary order parameter and, even when $\alpha_{02}>0$, allows $\mathrm{YMnO}_{3}$ to develop an (ionic) polarization when the $\mathrm{MnO}_{5}$ polyhedra have tilted and the $\mathrm{Y}-\mathrm{O}$ planes have buckled. The free energies of all typical improper ferroelectrics will contain such a term linear in $P$, although it may have a slightly different form. ${ }^{59}$ Since $P$ is no longer the primary order parameter, as shown in Fig. 2e, the conventional double-well minimum does not exist $\left(\alpha_{02}>0\right.$; in some nominally improper ferroelectrics the polarization may be unstable and produce a double-well minimum by itself, but the energy lowering will be minimal compared to that found for the polarization coupled to the primary order parameter). Rather, the free energy exhibits a single-well minimum shifted to a nonzero value of $P$ owing to the coupling term in which $-\beta_{31} R^{3}$ acts as an effective electric field that induces $P$ (Fig. 2f). We

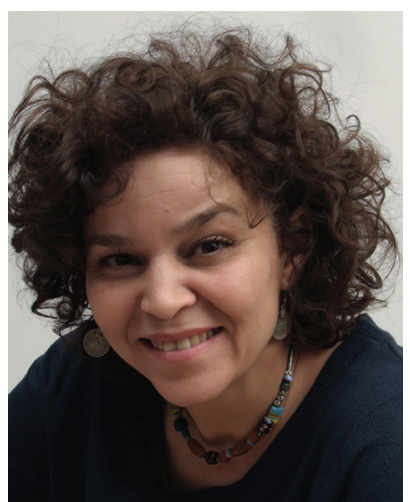

Hania Djani
Hania Djani graduated in Physics and Materials Sciences from Algiers University of Sciences and Technology Houari Boumedienne (USTHB) in Algeria. She received her $\mathrm{PhD}$ from the same University in 2013. She is a permanent researcher at the Center of Development of Advanced Technologies (CDTA) in Algiers. Her research interests mainly concern ferroelectric complex oxide structures, which she began to study experimentally, using PLD and magnetron PVD deposition techniques and then continued to investigate theoretically from firstprinciples calculations, after a two-years stay (2010-2012) at the University of Liège (Belgium) with Pr. Philippe Ghosez.

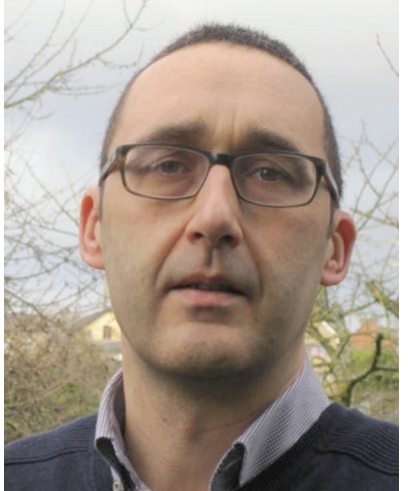

Philippe Ghosez
Philippe Ghosez graduated from Université Catholique de Louvain (Belgium) and undertook a Ph.D there under the supervision of $X$. Gonze and J.-P. Michenaud. He was a postdoctoral research associate at Yale University in the group of K. M. Rabe in 1998-1999. He is Professor at Université de Liège (Belgium) since 1999 and is currently also Francqui Research Professor. He has been involved in the first-principles theory and modeling of complex oxides for more than 20 years. 


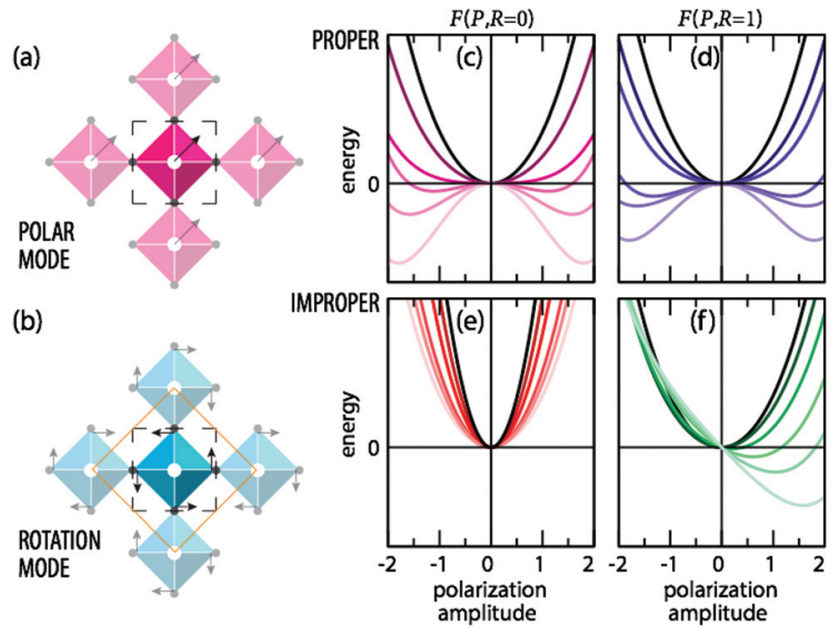

Fig. 2 Schematic energy contours reveal the change in the energy profile upon cooling (lighter colors correspond to lower temperatures) for (c) a proper and ( $f$ ) an improper ferroelectric transition driven by a non-polar mode $R$, such as the oxygen rotation mode shown in (b). Panels (c) and (e) and panels (d) and (f) show the polarization well in the absence $(R=0)$ and presence $(R=1)$ of the non-polar distortion $R$, respectively. In a proper ferroelectric, the coupling between the polarization and rotation is frequently bi-quadratic, and renormalizes the potential energy landscape such that the interaction leads to a less-pronounced double-well potential (d). The effect of the odd coupling of the polar and non-polar modes on the energy surface for an improper ferroelectric is shown in panel ( $f$ ), where the minimum shifts to a non-zero value of the polarization amplitude. Note that a symmetry-related energy profile exists, but is not depicted for ( $f$ ), with a parabola that has a minimum at an equivalent negative polarization amplitude.

also note that one convenient way of assessing unambiguously the improper ferroelectric behavior of a compound is from calculations under open-circuit electrical boundary conditions, ${ }^{52}$ which has recently been made possible with advances owing to the modern theory of electric polarization. ${ }^{60}$

Physically, the coupling term (odd in $P$ and $R$ ) indicates that when an electric field is applied to an improper ferroelectric, the primary order parameter will switch direction in addition to the polarization. As we discuss below, this has important consequences for the design of multifunctional materials. van

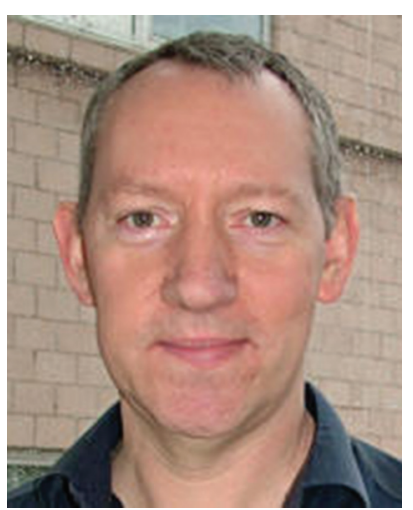

Philip Lightfoot
Philip Lightfoot graduated from Oxford University, and undertook a D.Phil there under the supervision of Tony Cheetham. He took up a position at St. Andrews in 1990, and is currently Professor of Solid State Chemistry. His interests encompass a wide range of functional solids; their synthesis, crystal structures and properties, with an emphasis on structure-property relations studied by diffraction methods.
Aken and co-workers used first-principles calculations to show that, unlike the ferroelectric transitions of $\mathrm{BaTiO}_{3}$ and $\mathrm{PbTiO}_{3}$, the transition in $\mathrm{YMnO}_{3}$ is accompanied by little to no charger transfer or rehybridization. They thus concluded that, "the mechanism is driven entirely by electrostatic and [ion] size effects, rather than the usual changes in chemical bonding associated with ferroelectric phase transitions in perovskite oxides." 56

Materials that undergo ferroelectric transitions (or more generally, transitions to a polar structure) through a trilinear coupling mechanism contain a term in their free energies in which the polarization is linearly coupled to two other nonpolar order parameters $R_{1}$ and $R_{2}$ viz., $\mathcal{F}=\gamma P R_{1} R_{2}$, where $\gamma$ is a coefficient and $R_{1}$ and $R_{2}$ are non-polar structural distortions of different symmetry. The origin of the term 'trilinear coupling' should now be apparent. This mechanism is somewhat peculiar since it does not appear to fulfill one of the basic postulates of Landau theory, that of a single order parameter. The question of which structural distortion $-P, R_{1}$ or $R_{2}-$ drives the transition and the order in which the phase transitions actually take place is somewhat complicated and differs for different materials. In the Aurivillius phase $\mathrm{SrBi}_{2} \mathrm{Nb}_{2} \mathrm{O}_{9}$, a polar mode (transforming like the irreducible representation $\mathrm{E}_{\mathrm{u}}$ ) and a zone-boundary mode $\left(\mathrm{X}_{3}{ }^{-}\right)$first condense simultaneously in a so-called avalanche transition ${ }^{49}$ (which is firstorder), accompanied by another zone-boundary mode $\left(\mathrm{X}_{2}^{+}\right)$ that is coupled to the first two and appears as a secondary order parameter. ${ }^{17,61}$ In this case, the primary order parameter actually consists of two different distortions, $\mathrm{E}_{\mathrm{u}}$ and $\mathrm{X}_{3}{ }^{-}$. The fact that both modes condense at the same temperature is highly unusual, since two lattice distortions of different symmetries would not be expected to have the same temperature dependence. Another possibility is when the primary order parameter consists of two non-polar (zone-boundary) lattice distortions, which in combination give rise to a macroscopic polarization. This scenario - nowadays known as "hybrid improper" ferroelectricity - was first proposed to be the origin of ferroelectricity in artificial $\mathrm{PbTiO}_{3} / \mathrm{SrTiO}_{3}$ superlattices, ${ }^{47} \mathrm{NaLaMnWO}_{6}$ double perovskites ${ }^{51}$ and a $\mathrm{Ca}_{3} \mathrm{Mn}_{2} \mathrm{O}_{7}$ Ruddlesden-Popper compound. ${ }^{50}$

In the remainder of this Perspective, we will highlight the manner in which the trilinear coupling mechanism gives rise to ferroelectricity or polar structures in different families of layered perovskites. In particular, we aim to show that this mechanism can provide a unifying framework for rationalizing the crystal chemistry of polar structures across many families of materials. We also highlight the possibility of using the trilinear coupling mechanism as a generic tool to design new multifunctional materials.

Before beginning our review of layered perovskites, we make a few notes here regarding terminology. First, we use the term 'ferroelectric' to describe a material that displays a spontaneous macroscopic polarization (that is, the material is polar), which may in principle be switched to a symmetry-equivalent state with an applied electric field. Materials for which polarization switching has been demonstrated experimentally 
will be noted explicitly. Second, we use the terms octahedral 'rotation' and 'tilt' interchangeably to mean the same thing: a structural distortion involving rotation of the $\mathrm{BO}_{6}$ octahedra about one or more crystallographic axes. Finally, although we will make frequent mention of various structural distortions, any discussions of phase transitions or critical behavior will be limited to experimental observations only, as detailed information concerning the dynamics of phase transitions is not really available from first-principles calculations.

\section{Trilinear coupling in action}

\subsection{Double perovskites and superlattices}

The early 2000s saw many efforts devoted to understanding the effects of dimensionality and finite-size on ferroelectricity in thin-films of various $\mathrm{ABO}_{3}$ perovskite materials. ${ }^{62-66}$ Initially, the focus was mostly on thin-films of a single material, playing with electrical ${ }^{67-71}$ and mechanical boundary conditions, ${ }^{72-76}$ but the interests of the community naturally evolved to artificial superlattices built from the repetition of two or more different $\mathrm{ABO}_{3}$ perovskite layers alternately stacked one over the other in a manner akin to Lego blocks. This includes so-called 'bicolor' superlattices such as $\mathrm{SrTiO}_{3} /$ $\mathrm{BaTiO}_{3}{ }^{42} \mathrm{SrTiO}_{3} / \mathrm{PbTiO}_{3}{ }^{43,44,47,77,78} \mathrm{KNbO}_{3} / \mathrm{KTaO}_{3}{ }^{45,46}$ and even tricolor systems, such as $\left(\mathrm{ABO}_{3}\right)_{l} /\left(\mathrm{A}^{\prime} \mathrm{BO}_{3}\right)_{m} /\left(\mathrm{A}^{\prime \prime} \mathrm{BO}_{3}\right)_{n}{ }^{79-81}$ that were shown to naturally break inversion symmetry. This activity gave rise to many fundamental discoveries fueled by the combination of first-principles calculations based on density functional theory and experimental investigations relying on advanced layer-by-layer growth deposition techniques and local probe measurements. ${ }^{62-66,82}$

Artificial superlattices appeared at first glance as merely a nice playground for finely tuning ferroelectric properties through appropriate control of layer thickness. For example, $\mathrm{SrTiO}_{3}$ is nominally not ferroelectric but undergoes a transition to a structure with octahedral rotations at $105 \mathrm{~K}$, whereas $\mathrm{PbTiO}_{3}$ is a good ferroelectric with no octahedral rotations. In $\mathrm{SrTiO}_{3} / \mathrm{PbTiO}_{3}$ superlattices the polarization and phase transition temperature can be tuned systematically and scale predictably with the $\mathrm{PbTiO}_{3}$ volume fraction. Hence, if the thickness of the $\mathrm{SrTiO}_{3}$ layers is held constant at 3 unit cells (as in ref. 43), the polarization increases as the $\mathrm{PbTiO}_{3}$ layer thickness increases, as expected. However, Dawber and co-workers ${ }^{43}$ made a surprising and intriguing discovery: the polarization decreases as the $\mathrm{PbTiO}_{3}$ layer thickness decreases, but re-appears unexpectedly when there are only one or two $\mathrm{PbTiO}_{3}$ unit cells in the $\mathrm{PbTiO}_{3}$ layer. Why does ferroelectricity re-appear in the limit of ultra-thin $\mathrm{PbTiO}_{3}$ layers, precisely where it is least favorable?

A superlattice consisting of alternating layers of undistorted $\mathrm{ABO}_{3}$ and $\mathrm{A}^{\prime} \mathrm{BO}_{3}$ has tetragonal $P 4 / m m m$ symmetry (one can think of such a superlattice as an A-site ordered double perovskite with cation order along [001]). Focusing on a $\mathrm{PbTiO}_{3} /$ $\mathrm{SrTiO}_{3}$ superlattice in which one layer of $\mathrm{PbTiO}_{3}$ alternates with one layer of $\mathrm{SrTiO}_{3}$, Bousquet et $a l^{47}$ showed that the lowest-energy structure has polar $\mathrm{P} 4 \mathrm{bm}$ symmetry and that this atomic structure can be reached from the $P 4 / \mathrm{mmm}$ structure via a combination of three different structural distortions: (i) a polar distortion (transforming like the irreducible representation $\Gamma_{3}^{-}$) involving displacements of the $\mathrm{Pb}, \mathrm{Sr}$ and $\mathrm{Ti}$ cations against the oxygens along [001] (ii) an octahedral tilting distortion $\left(\mathrm{M}_{4}{ }^{-}\right)$corresponding to the Glazer tilt pattern $a^{0} a^{0} c^{-}$, and (iii) a second octahedral tilting distortion $\left(\mathrm{M}_{2}^{+}\right)$ corresponding to the Glazer tilt pattern $a^{0} a^{0} c^{+}$. Using Landau theory and a group theoretical analysis, Bousquet and coworkers showed that there is a term in the free energy, $\mathcal{F}=P R_{1}$ $R_{2}$, where $P$ is the $\Gamma_{3}{ }^{-}$polar mode and $R_{1}$ and $R_{2}$ are the $\mathrm{M}_{4}{ }^{-}$ and $\mathrm{M}_{2}{ }^{+}$octahedral tilting distortions. As we discussed above, this means that if $R_{1}$ and $R_{2}$ are present (that is, if the structure contains the two octahedral tilting distortions) then a polarization will automatically appear because $P$ is coupled to $R_{1}$ and $R_{2}$ through the trilinear term. The conclusion was thus that this trilinear coupling is responsible for the re-appearance of the polarization and the improper ferroelectric behavior observed experimentally (linear temperature dependence of $P$ and absence of divergence of the dielectric constant at the phase transition). ${ }^{47}$

The $\mathrm{SrTiO}_{3}$ substrate on which the superlattices are grown imposes an epitaxial strain on the film such that the $P 4 \mathrm{bm}$ phase is only just stable. Under slightly greater tensile strain, a $P m c 2_{1}$ phase is preferred, ${ }^{4,83}$ which again can be reached from $P 4 / \mathrm{mmm}$ via a combination of three different structural distortions: (i) an octahedral tilting distortion corresponding to $a^{0} a^{0} c^{+}$, as above, (ii) an octahedral tilting distortion corresponding to $a^{-} a^{-} c^{0}\left(\mathrm{M}_{5}{ }^{-}\right)$and, (iii) a polar distortion transforming like the irrep ${\Gamma_{5}}^{-}$, which produces a polarization in the [110] direction, i.e. in the plane of the substrate, instead of perpendicular to it. Amazingly, the symmetry of these three modes is also compatible with a trilinear coupling term of exactly the same form as above. In contrast to the polar mode in the superlattices with $\mathrm{P} 4 \mathrm{bm}$ symmetry, here the crystal chemical origin of the polarization can be traced back to antipolar displacements of the A-site cations. The microscopics of this mechanism were elucidated on studies of both A-site ordered double perovskites and Ruddlesden-Popper phases, as described below.

Rondinelli and Fennie showed ${ }^{39}$ using symmetry arguments and first-principles calculations that the presence of two chemically distinct A-site cations in $\mathrm{AA}^{\prime} \mathrm{B}_{2} \mathrm{O}_{6}$ double perovskites with layered ordering along [001] (again, these systems can be thought of as $\left(\mathrm{ABO}_{3}\right)_{1} /\left(\mathrm{A}^{\prime} \mathrm{BO}_{3}\right)_{1}$ superlattices $)$ shifts the location of the inversion centers in the undistorted $P 4 / \mathrm{mmm}$ paraelectric structure such that a combination of two octahedral rotations can globally lift inversion symmetry and allow a macroscopic polarization to arise. Note that no combination of octahedral rotations can globally lift inversion symmetry in a bulk $\mathrm{ABO}_{3}$ perovskite. Hence, as for the superlattices above, the free energies of these double perovskites contain a trilinear term that couples the polarization to the two octahedral rotation modes. 
This understanding was used to formulate a set of design guidelines, whereby polar A-site ordered double perovskites were constructed by layering two non-polar Pnma perovskites; the Pnma structure is composed of the two tilt patterns $a^{0} a^{0} c^{+}$ and $a^{-} a^{-} c^{0}$, i.e., $a^{-} a^{-} c^{+}$(these rotations transform like the irreps $\mathrm{M}_{3}{ }^{+}$and $\mathrm{R}_{4}{ }^{+}$, respectively). These guidelines were stated in the form of a chemical criterion and an energetic criterion. The chemical criterion merely states that the A-sites of the double perovskite must be occupied by two chemically distinct cations. The energetic criterion requires that the two perovskites used to construct the layered material have a strong energetic tendency towards the $a^{-} a^{-} c^{+}$tilt pattern (subsequent work ${ }^{84}$ also showed that the layered ordering must be along the direction of the $c^{+}$tilt, or inversion symmetry lifting does not occur). The resulting double perovskite has polar $P m c 2_{1}$ symmetry.

The crystal chemical origin of the polarization in these double perovskites was identified by Mulder and co-workers ${ }^{40}$ as arising from anti-polar displacements of the A-site cations (transforming like the irrep $\mathrm{X}_{5}^{+}$). Not all octahedral rotation patterns allow the A-site to shift from its ideal position in the cubic perovskite structure. The $a^{-} a^{-} c^{+}$tilt pattern that produces the Pnma space group does allow such A-site displacements (along the [110] direction with respect to the cubic perovskite axes) and they in fact produce a polarization in the A-O layers of Pnma perovskites, as shown in Fig. 4a. However, the A-sites are related by inversion symmetry through the B-site and hence the induced polarizations are of exactly equal and opposite magnitude such that they cancel each other out. If there are two chemically distinct A-sites however, as in A-site ordered double perovskites, then the induced polarizations are no longer exactly equal and the cancelation is incomplete, leaving a residual macroscopic polarization (Fig. 4b). This makes such materials ferrielectrics, or electronic analogues of ferrimagnets. ${ }^{85}$

What determines the magnitude of the polarization in these A-site ordered double perovskites? The A-site cation displacements are coupled to the octahedral rotations such that the larger the magnitude of the rotations, the larger the A-site cation displacements. One might then naïvely expect that the polarization should be maximized for double perovskites built from components with large octahedral rotation distortions. However, if both $\mathrm{ABO}_{3}$ and $\mathrm{A}^{\prime} \mathrm{BO}_{3}$ have large octahedral rotations, then the A-site cation displacements will be similar in magnitude and though they may not completely cancel (and the A and A'Born effective charges may be slightly different), the total polarization will be small. In addition, the barrier to switch the polarization will be large. A better strategy, outlined here as a simplified version of the theory presented in ref. 40 is to select $\mathrm{ABO}_{3}$ and $\mathrm{A}^{\prime} \mathrm{BO}_{3}$ such that "the average tolerance factor is maximized and the difference in their tolerance factors is also large." ${ }^{40}$ In other words, the difference in the tendency of the A-site cations to displace will be maximized (if A prefers to displace very little, while the $\mathrm{A}^{\prime}$ cation displaces a lot), leading to a minimal cancelation of layer polarizations, a large total polarization and a smaller switching barrier. The stannate $\mathrm{ASnO}_{3} / \mathrm{A}^{\prime} \mathrm{SnO}_{3}$ superlattices $\left(\mathrm{A}, \mathrm{A}^{\prime}=\mathrm{Ca}, \mathrm{Sr}\right.$, and $\left.\mathrm{Ba}\right)$ are predicted to best satisfy these design guidelines; ${ }^{86}$ however, experimental realization of the system remains to be reported. The design rules also allow for the design and prediction of the net polarization in $\left(\mathrm{ABO}_{3}\right)_{m} /\left(\mathrm{A}^{\prime} \mathrm{BO}_{3}\right)_{n}$ superlattices of arbitrary thickness. Most importantly, for a given $\mathrm{A}$ and $\mathrm{A}^{\prime}$, the polarization is maximized if both $m$ and $n$ are odd (and it will be exactly zero if both $m$ and $n$ are even). Another key outcome from these analyses is that the size of the polarization is directly proportional to the degree of A-site ordering; hence, the maximum polarization is obtained when the A-site cations are completely ordered with a square-wave like composition variation along the [001] direction. Bellaiche and Íñiguez have also shown that the coupling between the anti-polar A cation displacements and octahedral tilt modes is a general feature in perovskite oxides due to induced interatomic forces. ${ }^{53,87}$ Interestingly, if a proper ferroelectric is used as a building block in these superlattices, e.g., as in $\mathrm{BaTiO}_{3} / \mathrm{CaTiO}_{3}$, then a proper out-of-plane polarization results that reduces the $P m c 2_{1}$ symmetry to $P c .{ }^{88}$

Since inversion symmetry is broken in the layered $1 / 1$ superlattices by the combination of two octahedral rotation modes, such oxides can readily exhibit long-range magnetic order by selecting transition metal B cations with open d-shell configurations. One of the first multiferroic systems proposed to fulfill these conditions includes the $\left(\mathrm{BiFeO}_{3}\right)_{1} /\left(\mathrm{LaFeO}_{3}\right)_{1}$ superlattice epitaxially grown on a (001)- $\mathrm{SrTiO}_{3}$ substrate. ${ }^{89}$ Although, the thermodynamically stable phase of $\mathrm{BiFeO}_{3}$ is a rhombohedral $R 3 c$ structure with the $a^{-} a^{-} a^{-}$tilt system, and thus does not immediately satisfy the guidelines provided by Rondinelli and Fennie, it does have a strong tendency to the Pnma-tilt distortions. ${ }^{90}$ In contrast, $\mathrm{LaFeO}_{3}$ does exhibit the $a^{-} a^{-} c^{+}$tilt at room temperature. Both compounds are also G-type antiferromagnets with Neél temperatures well-above room temperature. First-principles calculations found that the equilibrium structure of the ferrate superlattice is indeed polar, $P m c 2_{1}$, owing to trilinear coupling among the octahedral tilt and polar modes with a sizable polarization of $11.6 \mu \mathrm{C} \mathrm{cm}^{-2}$ along the [110] direction. Because the magnetic spins of the $\mathrm{B}$ cations in orthorhombic perovskites are not required to be collinear, ${ }^{91} \mathrm{a}$ small spin canting can support a net magnetization in the AFM phases. In this case, the easy axis for the spins was computed to be along the [011] direction, which is perpendicular to the direction of the net polarization. The net spin-canted moment of $\sim 0.40 \mu_{\mathrm{B}}$ is induced by the polar displacements and aligned along the $[00 \overline{1}]$ direction, i.e., orthogonal to both the polarization and the easy magnetization axis. The sign of the canted moment is controlled by the $a^{-} a^{-} c^{0}$ tilt, and electric switching the direction of the weak magnetization requires reversing both the polarization direction and sense of the outof-phase tilts. It was proposed that in-plane rotation of the polarization might be a possible low-energy switching path allowing this. ${ }^{89}$

This concept was extended to other $\left(\mathrm{LaFeO}_{3}\right)_{n} /(\mathrm{LnFeO})_{m}(\mathrm{Ln}=$ lanthanide) ferrate ${ }^{92}$ and the $\left(\mathrm{LaCrO}_{3}\right)_{n} /\left(\mathrm{YCrO}_{3}\right)_{m}$ chromate ${ }^{53}$ superlattices with odd periodicities. Remarkably, electronic- 
structure calculations on the ferrates demonstrate that electrical switching of magnetization should be feasible through the coupling of multiple lattice modes with weak ferromagnetism, circumventing the complications with finding single phase materials with coexisting ferroic orders that originate from conventional mechanisms. In fact, the magnetoelectric response in the $\left(\mathrm{LaFeO}_{3}\right)_{1} /\left(\mathrm{YFeO}_{3}\right)_{1}$ superlattice was found to be two-to-three times larger than that of the prototypical magnetoelectric $\mathrm{Cr}_{2} \mathrm{O}_{3}$. Recently these first-principles derived guidelines have been realized in the [001] ordered odd period superlattice $\left(\mathrm{LaFeO}_{3}\right)_{5} /\left(\mathrm{YFeO}_{3}\right)_{5}$ grown using pulsed-laser deposition. ${ }^{93}$ Second-harmonic generation polarimetry plots indicate the film is polar (point group $\mathrm{mm} 2$ consistent with the theoretical predictions), and room temperature magneto-optical Kerr experiments reveal long-range order consistent with the bulk components, but the weak-ferromagnetism remains to be reported. Although the superlattice was not digitally ordered a net polar structure results from the uncompensated layer polarizations as predicted by Mulder et al. $^{40}$

Generalization of the design guidelines discussed above shows that layering of both A and B sites along additional directions besides [001] makes it possible to lift inversion symmetry with octahedral rotations. Fig. 3 shows the multitude of simple orderings possible in double perovskites; many more may be accessible using non-equilibrium thin film growth methods. ${ }^{94,95}$ The manner in which inversion can be removed depends on the details of the octahedral tilt system, and here we describe some of those most promising order schemes, which should be experimentally accessible owing to coordination-driven cation order.

Ordering of cations along the [111]-direction as in $\mathrm{AA}^{\prime} \mathrm{B}_{2} \mathrm{O}_{6}$ perovskites and depicted in Fig. 3c, requires tilts consisting of two modes (such as $a^{-} a^{-} c^{+}$) as already described, ${ }^{41,96}$ or alternatively a single mode as recently pointed out by Young and Rondinelli. ${ }^{97}$ In the latter case, only out-of-phase rotations are required: first-principles calculations on a series of aluminates, including $\mathrm{LaNdAl}_{2} \mathrm{O}_{6}, \mathrm{LaPrAl}_{2} \mathrm{O}_{6}$, and $\mathrm{CePrAl}_{2} \mathrm{O}_{6}$, found that while only $\mathrm{LaPrAl}_{2} \mathrm{O}_{6}$ and $\mathrm{CePrAl}_{2} \mathrm{O}_{6}$ are polar (Imm2), $\mathrm{LaNdAl}_{2} \mathrm{O}_{6}$ is chiral and non-polar (space group R32). Interestingly, all are predicted to undergo a transition to the $R 32$ chiral phase at higher temperatures, which possesses relatively large piezoelectric coefficients, comparable to those of common lead-free piezoelectric materials such as $\mathrm{BaTiO}_{3}$ and $\mathrm{LiNbO}_{3}$. Unlike the Pnma orthorhombic perovskites, inversion symmetry in the rhombohedral aluminates with tendencies to only out-of-phase rotations results from coupling between a single tilt mode and the 'polar' displacements. Comparing the polarization of $\mathrm{AA}^{\prime} \mathrm{B}_{2} \mathrm{O}_{6}$ with [001] and [111] ordering and $a^{-} a^{-} c^{+}$, the former are always found to exhibit larger polarizations Interestingly, if the $\mathrm{A}$ cations are ordered along ${ }^{110}$ (Fig. 3b), there is no combination of octahedral rotations that will lift inversion symmetry. This was shown using representation theory and supported by first-principles calculations on gallate, zirconate, and hafnate superlattices ${ }^{97}$ (note that if B cation ordering is included, this restriction is lifted). (a)

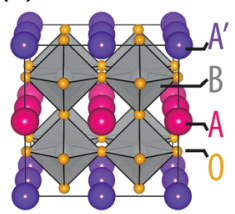

(d)

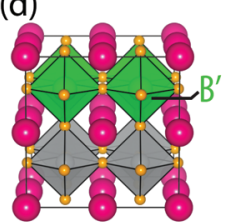

(b)

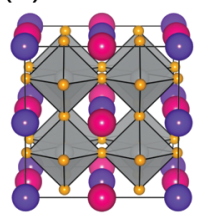

(e)

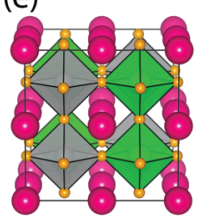

(c)

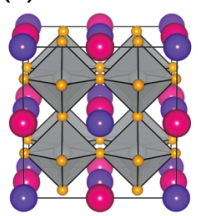

(f)

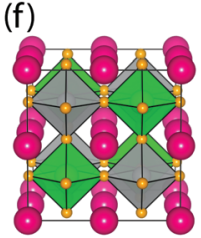

(g)

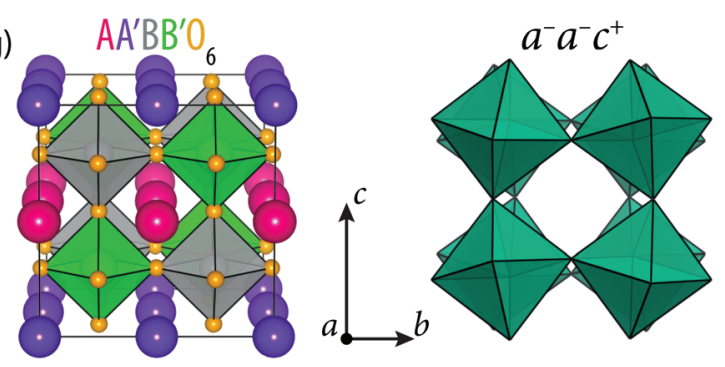

Fig. 3 Cation ordered variants of double perovskite without octahedral distortions: $\mathrm{AA}^{\prime} \mathrm{B}_{2} \mathrm{O}_{6}$ with (a) layered, (b) columnar, (c) rock-salt ordering of $A$ and $A^{\prime}$ cations, and $A_{2} B^{\prime} O_{6}$ (d) layered, (e) columnar, (f) rock-salt ordering of $B$ and $B^{\prime}$ cations. ( $g$ ) The common ordering adopted by $A A^{\prime}$ $\mathrm{BB}^{\prime} \mathrm{O}_{6}$ double perovskites (left) owing to valence preferences and the requirement to alleviate under and over-coordinated cations. The layered A-site order with rock salt B-site order and the orthorhombic tilt pattern (right) leads results in a polar-chiral space group $P 2_{1}$.
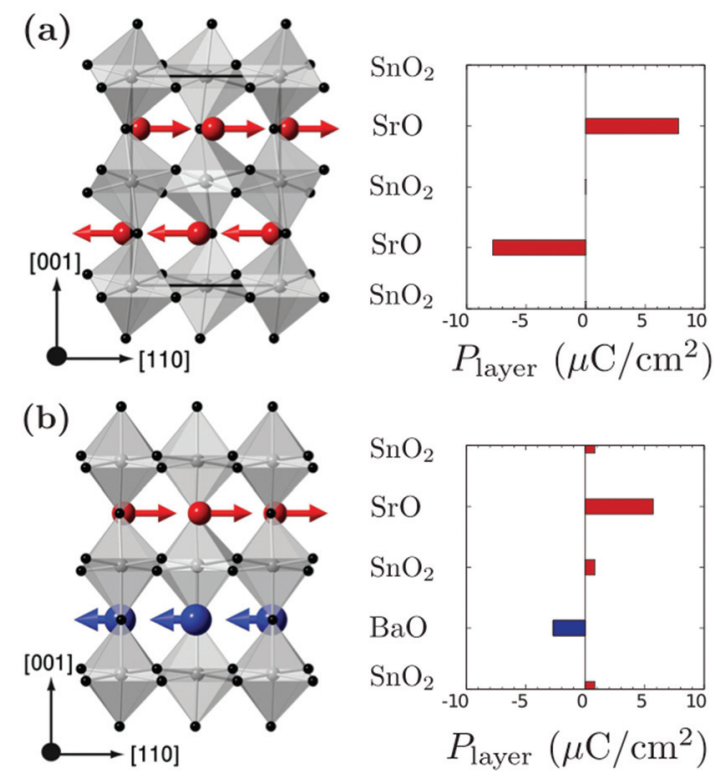

Fig. 4 (a) Layer-resolved polarization of $\mathrm{Pnma}_{\mathrm{SrSnO}}$ and (b) $\mathrm{BaSrSn}_{2} \mathrm{O}_{6}$ double perovskite (in polar space group $\mathrm{Pmc2}_{1}$ ) from firstprinciples calculations. ${ }^{40}$ Notice that the polarizations induced in the $\mathrm{Sr}-\mathrm{O}$ layers in $\mathrm{SrSnO}_{3}$ are exactly equal and opposite and hence cancel each other out such that the macroscopic polarization is zero. This cancelation is incomplete in the double perovskite, and a residual polarization remains. 
Double perovskites with the $\mathrm{AA}^{\prime} \mathrm{BB}^{\prime} \mathrm{O}_{6}$ stoichiometry tend to exhibit layered ordering of the $\mathrm{A}$ and $\mathrm{A}^{\prime}$ cations and rocksalt ordering of the B and B' (Fig. 3g), because these arrangements best optimize the electrostatic interactions. ${ }^{37}$ Examples of such compounds include $\mathrm{NaLaMgWO}_{6}$ or $\mathrm{NaLaScNbO}_{6}$. A magnetic version of the former, $\mathrm{NaLaMnWO}_{6}$, was first reported in 2009 by Woodward and co-workers ${ }^{98}$ to be polar $\left(P 2_{1}\right)$, and subsequently first-principles calculations elucidated the atomistic origin of the inversion symmetry to arise from the coupling of the octahedral tilt modes in the presence of the cation order. ${ }^{51}$ Recent experimental study, however, found no spontaneous electric polarizations in samples of $\mathrm{NaLaMnWO}_{6}$ and $\mathrm{NaNdMnWO}_{6}$, which was attributed to difficulty in making dense ceramics required for electrical poling measurements. ${ }^{99}$ The design of new multiferroics based on this cation-ordering concept was extended to double perovskites with the same cation order on the $\mathrm{A}$ and $\mathrm{B}$ sites, but included magnetic $\mathrm{Ni}$ and $\mathrm{Mn}$ cations on the B-site to stabilize ferromagnetic order. ${ }^{100}$ (Recall that the double order and $a^{-} a^{-} c^{+}$tilt is sufficient to provide for an electric polarization, yet if only B-site order is present then the structure is non-polar $P 2_{1} / n$, consistent with the guidelines of Rondinelli and Fennie). In the $\mathrm{RLaNiMnO}_{6}$ perovskites, where $\mathrm{R}$ is a rare-earth ion, the in-plane polarization increases as the difference in ionic radius between the $\mathrm{R}$ and La cation increases owing to the change in the octahedral tilts: for example, CeLaNiMnO 6 $\left(1.4 \mu \mathrm{C} \mathrm{cm}^{-2}\right)$ to ErLaNiMnO $_{6}\left(9.2 \mu \mathrm{C} \mathrm{cm}^{-2}\right)$ and this dependence is essentially the same as that attributed to tolerance factor arguments by Mulder and co-workers. In the theoretical studies, the magnetic ordering temperature for $\mathrm{CeLaNiMnO}_{6}$ is predicted to be close to room-temperature, and highly tunable with chemical substitution or epitaxial strain owing to the magnetostructural coupling found in the parent $R_{2}$ $\mathrm{NiMnO}_{6}$ compounds. ${ }^{101}$

\subsection{Ruddlesden-Popper phases}

Ruddlesden-Popper phases form a homologous series with general formula $\mathrm{A}_{n+1} \mathrm{~B}_{n} \mathrm{O}_{3 n+1}$. The Ruddlesden-Popper structure is generally described as a stacking of perovskite blocks along [001] (with respect to the cubic perovskite axes), with an extra rocksalt $\mathrm{AO}$ layer inserted every $n$ perovskite unit cells. Hence, in contrast to the $\mathrm{ABO}_{3}$ perovskite structure and the double perovskites and superlattice discussed above, the $\mathrm{BO}_{6}$ octahedra in Ruddlesden-Popper materials are continuously connected in only two dimensions (parallel to the $a$ and $b$ axes). This lattice topology - $\mathrm{BO}_{6}$ octahedra connected in only two dimensions versus three - is directly responsible for many of the functional properties of Ruddlesden-Popper phases, including fast low-temperature oxide ion mobility, ${ }^{102,103}$ superconductivity, ${ }^{104,105}$ and ferroelectricity induced by rotations of the $\mathrm{BO}_{6}$ octahedra. ${ }^{39,40,50}$ Among oxide Ruddlesden-Popper phases containing a single A-site cation, the A-site can be occupied by cations with valences of both $2+$ and $3+\left(\mathrm{Ba}^{2+}, \mathrm{Sr}^{2+}\right.$, $\mathrm{Ca}^{2+}$, rare earth cations) while the B-site is usually a transition metal with $2+$ or $4+$ valence (for example, $\mathrm{Ti}^{4+}, \mathrm{Ni}^{2+}, \mathrm{Mn}^{4+}$ or $\left.\mathrm{Mn}^{2+}, \mathrm{Ru}^{4+}\right)$. Note that in the case of Ruddlesden-Popper phases built from $\mathrm{A}^{3+} \mathrm{B}^{3+} \mathrm{O}_{3}$ perovskites, the valence state of the $\mathrm{B}$-site cation varies with $n$, e.g. the valence state of $\mathrm{Ni}$ in $\mathrm{La}_{2} \mathrm{NiO}_{4}(n=1)$ is formally $2+$, whereas it is $2.5+$ in $\mathrm{La}_{3} \mathrm{Ni}_{2} \mathrm{O}_{7}$ $(n=2)$. Nowadays, Ruddlesden-Popper phases can be epitaxially grown on perovskite substrates, with perfect control of the perovskite block thickness $n$, also opening the way to epitaxial strain engineering in this class of compounds. ${ }^{94,95}$

The mechanism by which ferroelectricity arises in Ruddlesden-Popper phases was first explained for $n=2 \mathrm{Ca}_{3} \mathrm{Ti}_{2} \mathrm{O}_{7}{ }^{25}$ and $\mathrm{Ca}_{3} \mathrm{Mn}_{2} \mathrm{O}_{7}{ }^{26,27}$ Neither of the parent perovskites of these materials $\left(\mathrm{CaTiO}_{3}\right.$ and $\left.\mathrm{CaMnO}_{3}\right)$ are polar in bulk, forming instead in the non-polar Pnma space group. $\mathrm{Ca}_{3} \mathrm{Ti}_{2} \mathrm{O}_{7}$ has only been reported in the polar $A 2{ }_{1}$ am space group whereas $\mathrm{Ca}_{3} \mathrm{Mn}_{2} \mathrm{O}_{7}$ undergoes a structural phase transition from a nonpolar tetragonal $I 4 / \mathrm{mmm}$ phase to $A 2_{1} \mathrm{am}$ in the range 200-300 ${ }^{\circ} \mathrm{C}^{27}$ Benedek and Fennie $^{50}$ showed that for both materials, the polar $A 2{ }_{1}$ am phase could be reached from I4/ $\mathrm{mmm}$ by a combination of octahedral rotation distortions corresponding to $a^{0} a^{0} c^{+}$(this distortion transforms like the irrep $\left.\mathrm{X}_{2}^{+}\right)$and $a^{-} a^{-} c^{0}\left(\mathrm{X}_{3}{ }^{-}\right)$. Both modes combined again give the familiar $a^{-} a^{-} c^{+}$tilt pattern of Pnma perovskites and couple trilinearly to a zone-center polar mode of $\Gamma_{5}{ }^{-}$symmetry. Hence, the Ruddlesden-Popper phases 'inherit' the structural distortions of their parent perovskites, like the $\mathrm{AA}^{\prime} \mathrm{B}_{2} \mathrm{O}_{6}$ family discussed above, and despite any differences in lattice topology between these two families of materials, the microscopic mechanism that gives rise to the polarization in $\mathrm{Ca}_{3} \mathrm{Ti}_{2} \mathrm{O}_{7}$ and $\mathrm{Ca}_{3} \mathrm{Mn}_{2} \mathrm{O}_{7}$ is identical to the double perovskites.

In an exciting development, Oh and co-workers ${ }^{106}$ recently demonstrated that the polarization in $\mathrm{Ca}_{3} \mathrm{Ti}_{2} \mathrm{O}_{7}$ single crystals can be switched with an applied electric field (although switching was not demonstrated, experimental support for the hybrid improper mechanism was also provided by Senn et $\left.a .^{107}\right)$. The work of oh et al. is significant for several reasons. Firstly, it was not apparent from the theoretical studies ${ }^{40,50}$ whether the polarization could be switched experimentally or what the switching mechanism might be. In particular, there were concerns that the switching barrier (and hence the switching field) may be impracticably high. Related to this, one of the most intriguing features of hybrid improper ferroelectricity is the possibility of coupling other lattice distortions or physical properties to the polarization, such that when the polarization is switched with an electric field, the other structural distortions or properties are switched also. For example, Benedek and Fennie showed ${ }^{50}$ that the magnetism in $\mathrm{Ca}_{3} \mathrm{Mn}_{2} \mathrm{O}_{7}$ is coupled to the polarization such that (a) when inversion symmetry is broken and the polarization condenses, the magnetization also switches on, and (b) when an electric field is used to switch the polarization, the magnetization also switches $180^{\circ}$. That is, the magnetization is electric fieldcontrollable. Although this prediction has not been confirmed experimentally, recent work by Pitcher et al. ${ }^{108}$ has shown that the polarization and magnetization can co-exist in double perovskite $\left(\mathrm{Ca}_{y} \mathrm{Sr}_{1-y}\right)_{1.15} \mathrm{~Tb}_{1.85} \mathrm{Fe}_{2} \mathrm{O}_{7}$ at room temperature, whereas $\mathrm{Ca}_{3} \mathrm{Mn}_{2} \mathrm{O}_{7}$ is only magnetically ordered below $115 \mathrm{~K}$; the polarization in this double perovskite compound arises 

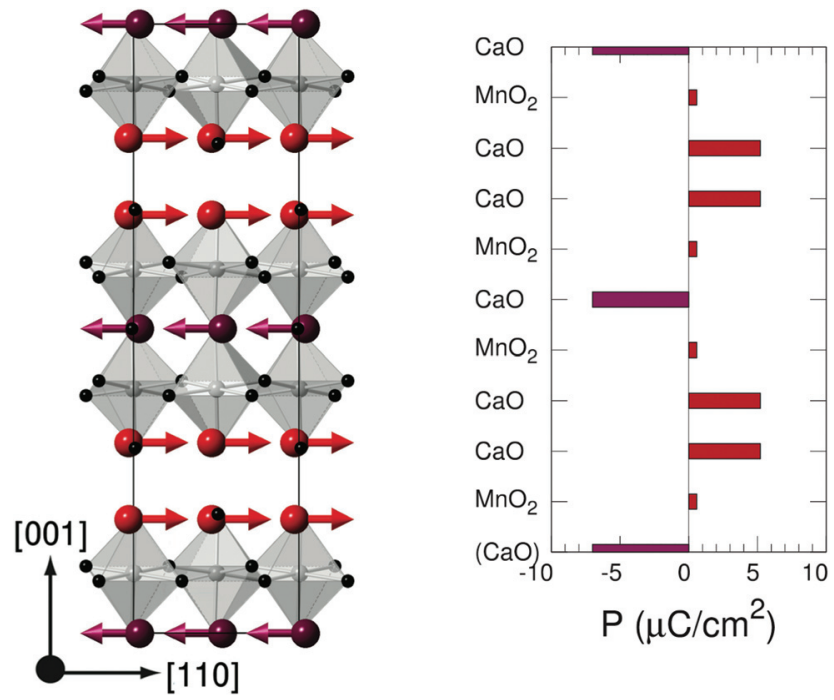

Fig. 5 Layer-resolved polarization of Ruddlesden-Popper $\mathrm{Ca}_{3} \mathrm{Mn}_{2} \mathrm{O}_{7}$ calculated from first principles. ${ }^{109}$ Notice that the two crystallographically distinct $\mathrm{A}$-sites give rise to layer polarizations that are oppositely oriented but not exactly equal, thus allowing for a residual macroscopic polarization.

through a trilinear coupling mechanism. We hope the very exciting and encouraging work of $\mathrm{Oh}$ and Pitcher will spur a search for truly field-controllable magnetism in other systems.

Fig. 5 shows the layer-resolved polarization for $\mathrm{Ca}_{3} \mathrm{Mn}_{2} \mathrm{O}_{7}$ from first-principles calculations. ${ }^{109}$ As in the double perovskites, the polarization arises from anti-polar displacements of the A-site cations. The rocksalt interface breaks the inversion center at the B-site such that the A-site cations in the AO layer directly adjacent to the interface are crystallographically different to the A-site cations in the middle of the perovskite block. Hence, the rocksalt interface satisfies the chemical criterion by creating symmetry-inequivalent A-sites. In addition, the interface breaks the connectivity of the $\mathrm{BO}_{6}$ octahedra along [001] such that there is an odd number of AO layers in the perovskite block, which ensures that the induced layer polarizations do not completely cancel. It follows that only even- $n$ Ruddlesden-Popper phases will be polar (through this mechanism), since only even- $n$ phases contain an odd number of AO layers in the perovskite block.

The relevant design criterion for Ruddlesden-Popper phases containing only a single A-site cation relies only on the tolerance factor of the parent $\mathrm{ABO}_{3}$ phase: as the tolerance factor decreases, the total polarization of the RuddlesdenPopper phases increases. ${ }^{40}$ However, the barrier to switch the polarization also increases. As in the cation ordered perovskites, ordering multiple cations can be used to circumvent this limitation. In fact, ref. 40 showed that the same general design guide - maximizing the tolerance factor mismatch and increase the average tolerance factor - may be applied to reduce this barrier in both $n=1$ and 2 Ruddlesden-Popper phases.
Recent work by Balachandran and co-workers ${ }^{110}$ has also provided a more exhaustive set of guidelines for lifting inversion symmetry in $n=1$ Ruddlesden-Popper phases, which tend to form in centosymmetric structures compared to the $n=2$ family. Three distinct cases were discussed: (i) no cation order, (ii), A-site order, and (iii) B-site order. The consequence of the cation ordering in combination with various octahedral tilt patterns, including those beyond the most familiar $a^{-} a^{-} c^{+}$, was then treated with group-theoretical methods. Interestingly and unlike the three-dimensional perovskites, there are combinations of tilt patterns that will lift inversion symmetry in the absence of cation order in $\mathrm{A}_{2} \mathrm{BO}_{4}$ materials (case i). The synthetic challenge, however, is choosing the optimal cations that will simultaneously favor two distinct tilt modes. In ref. 110 the authors provided a data-driven model founded on Bayesian inference that allows for the chemical selections. Case ii with A and $\mathrm{A}^{\prime}$ was also suggested to be a promising strategy and realized experimentally by Akamatsu et al. ${ }^{111}$ in a new $\mathrm{RNaTiO}_{4}$ family of piezoactive $n=1$ Ruddlesden-Popper phases. These experimental findings increased considerably the number of known $n=1$ Ruddlesden-Popper materials and demonstrate the power of combining predictive theories based on symmetry arguments with first-principles calculations and dedicated experimentation.

\subsection{Aurivillius phases}

Aurivillius phases of generic formula $\mathrm{Bi}_{2} \mathrm{~A}_{m-1} \mathrm{~B}_{m} \mathrm{O}_{3 m+3}$ form another interesting family of naturally-occuring layered perovskites. ${ }^{14}$ Their structure is made of fluorite-like $\mathrm{Bi}_{2} \mathrm{O}_{2}$ layers, alternating along the $c$-axis with perovskite-like $\mathrm{A}_{m-1} \mathrm{~B}_{m} \mathrm{O}_{3 m+1}$ blocks, where $m$ is the number of $\mathrm{BO}_{6}$ octahedra in the perovskite-like blocks; see Fig. 1. As in the Ruddlesden-Popper phases, the $\mathrm{BO}_{6}$ octahedra are only connected in two directions. Typically, the A-site (12-coordinated) is occupied by mono-, di- or trivalent cations such as $\mathrm{Na}^{+}, \mathrm{K}^{+}, \mathrm{Ba}^{2+}, \mathrm{Sr}^{2+}, \mathrm{Ca}^{2+}$, $\mathrm{Pb}^{2+}, \mathrm{Bi}^{3+}$ or $\mathrm{Ln}^{3+}$, and the B-site (6-coordinated) by $\mathrm{d}^{0}$ tetra-, penta- or hexavalent cations such as $\mathrm{Ti}^{4+}, \mathrm{Nb}^{5+}, \mathrm{Ta}^{5+}, \mathrm{W}^{6+}$. The case of non- $\mathrm{d}^{0}$ cations like $\mathrm{Fe}^{3+}, \mathrm{Ru}^{4+}, \mathrm{Cr}^{3+}, \mathrm{Ir}^{4+}$ or $\mathrm{Mn}^{4+}$ at the $\mathrm{B}$ site has also been considered in the search for magnetic and multiferroic Aurivillius phases. ${ }^{112-116}$

At the structural and functional levels, most Aurivillius phases share common characteristics. At high temperatures, they crystallize in the tetragonal $14 / \mathrm{mmm}$ space group (except for $\mathrm{Bi}_{2} \mathrm{WO}_{6}$, see later), which can be seen as the prototypical high-symmetry reference structure for the whole family. At low temperatures, the majority of Aurivillius phases adopt a polar orthorhombic (or monoclinic) ground-state with $a$ and $b$ cell parameters remaining very close. Typically, this ground-state is ferroelectric (except for $\mathrm{Bi}_{2} \mathrm{~W}_{2} \mathrm{O}_{9}{ }^{117}$ and $\mathrm{Sb}_{2} \mathrm{WO}_{6}{ }^{118}$ ) and exhibits a large spontaneous polarization $\left(\approx 30-50 \mu \mathrm{C} \mathrm{cm}{ }^{-2}\right)$ and high Curie temperature $\left(T_{\mathrm{C}}>600 \mathrm{~K}\right)$. Combined with fatiguefree behavior and low leakage currents, these features make these compounds very attractive for ferroelectric applications. They usually behave as normal ferroelectrics but systems with large atomic disorder between the $\mathrm{Bi}$ and A sites can also show relaxor behavior. ${ }^{119}$ The other functional properties (piezoelec- 
tric, electro-optics, etc.) of Aurivillius phases have not been extensively investigated so far but have been predicted to be comparable to conventional ferroelectrics, ${ }^{21}$ such as $\mathrm{PbTiO}_{3}$. Aurivillius phases appear attractive also in view of their high ionic conductivity ${ }^{120}$ and photocatalytic activity. ${ }^{121}$ They were also proposed as potential candidates for superconductivity. ${ }^{122}$

The experimentally observed orthorhombic or monoclinic ground-state structures result always from only small distortions of the $14 / \mathrm{mmm}$ reference phase. Their symmetry is, in each case, a subgroup of $14 / \mathrm{mmm}$ but not an isotropy subgroup, which means that modes transforming like more than one irreducible representation must be invoked to explain the observed symmetry breaking. The parent $14 / \mathrm{mmm}$ phase of these compounds typically exhibits numerous unstable phonon modes and the numbers and types of atomic motions involved in the symmetry lowering leading to the ground state can depend on the chemical composition but usually they include (i) tilts of the oxygen octahedra around the $a$-axis, (ii) rotations of the oxygen octahedra around the $c$-axis and (iii) polar cation motions along the $a$-axis, i.e., the (110) direction of the tetragonal reference structure. Recent studies shed new light on the way these distortions couple together to produce the ground state. In addition, in contrast to the double perovskites and Ruddlesden-Popper phases discussed above, the lattice dynamics of the Aurivillius phases cannot be linked to any parent perovskite phase. This is because the thickness $m$ of the perovskite-like block cannot be systematically increased while keeping the same A and B cations. The only exception is in the absence of $\mathrm{A}$ cations, as in the $\mathrm{Bi}_{2} \mathrm{~W}_{m} \mathrm{O}_{3 m+3}$ series. The reason originates in the formal charges of the $\left[\mathrm{Bi}_{2} \mathrm{O}_{2}\right]^{2+}$ and $\left[\mathrm{A}_{m-1} \mathrm{~B}_{m} \mathrm{O}_{3 m+1}\right]^{2-}$ layers which requires the combination of $\mathrm{A}^{x+}$ and $\mathrm{B}^{y+}$ cations satisfying: $(m-1) x+m y-2(3 m-1)=-2$ or equivalently $(x+y-6) m=x$. For $m=1$, the only possibility is $x=0$ and $y=6$, which appears also as a generic solution for any value of $m$. At $m=2$ and $m=3$, another integer solution is $(x=2, y=5)$ and $(x=3, y=4)$ respectively. Those with larger $m$ typically combine different cations at either A or B site, in order to provide an appropriate average fractional formal charge. As illustrated in Table 1, various phases are known and have been investigated extensively. ${ }^{15,22,123-129}$ Aurivillius can also form mixed layered structures in which $\mathrm{Bi}_{2} \mathrm{O}_{2}$ planes alternates with two or more perovskite-like blocks of different thicknesses and composition. Examples of such intergrowths are $\mathrm{Bi}_{7} \mathrm{Ti}_{4} \mathrm{NbO}_{21}$ which can be viewed as the stacking $\mathrm{Bi}_{3} \mathrm{TiNbO}_{9}(m=2)$ and $\mathrm{Bi}_{4} \mathrm{Ti}_{3} \mathrm{O}_{12}(m=3)^{130}$ or $\mathrm{Bi}_{10} \mathrm{Ti}_{3} \mathrm{~W}_{3} \mathrm{O}_{30}$ combining $\mathrm{Bi}_{4} \mathrm{Ti}_{3} \mathrm{O}_{12}(m=3)$ with $\mathrm{Bi}_{2} \mathrm{WO}_{6}(m=1) .{ }^{131}$

$\mathrm{Bi}_{2} \mathrm{WO}_{6}(m=1, \mathrm{BWO})$ is unique amongst the Aurivillius phases in that it adopts, at high temperatures, an unusual A2/ $m$ paraelectric monoclinic phase with edge-shared oxygen octahedra. On cooling, it exhibits a phase transition at $950{ }^{\circ} \mathrm{C}$ to an intermediate ferroelectric phase of $B 2 c b$ symmetry and then a second transition at $670{ }^{\circ} \mathrm{C}$ to a ground-state ferroelectric phase of $P 2_{1} a b$ symmetry. Both these ferroelectric phases appear as small distortions of the aristotype $14 / \mathrm{mmm}$ phase, although this latter is never reached at high temperature (the system instead undergoes a first-order transition to the $A 2 / m$
Table 1 Examples of $\mathrm{Bi}_{2}{ }^{3+} \mathrm{A}_{m-1}^{x+} \mathrm{B}_{m}^{y+} \mathrm{O}_{3 m+3}^{2-}$ Aurivillius compounds in terms of the perovskite block thickness $m$ and formal charges $x$ and $y$ of the $A$ and $B$ cations

\begin{tabular}{lllll}
\hline$m$ & $x$ & $y$ & Example & Ref. \\
\hline 1 & - & 6 & $\mathrm{Bi}_{2} \mathrm{WO}_{6}, \mathrm{Bi}_{2} \mathrm{MoO}_{6}$ & $118,126,128,132$ \\
2 & - & 6 & $\mathrm{Bi}_{2} \mathrm{~W}_{2} \mathrm{O}_{9}$ & 117 \\
& 2 & 5 & $\mathrm{CaBi}_{2} \mathrm{Nb}_{2} \mathrm{O}_{9}, \mathrm{SrBi}_{2} \mathrm{Ta}_{2} \mathrm{O}_{9}$ & $15,125,127,133$ \\
3 & - & 6 & $\mathrm{Bi}_{2} \mathrm{~W}_{3} \mathrm{O}_{12}$ & 134 \\
& 3 & 4 & $\mathrm{Bi}_{4} \mathrm{Ti}_{3} \mathrm{O}_{12}$ & $22,123,124,127$ \\
4 & - & 6 & $\mathrm{Bi}_{2} \mathrm{~W}_{4} \mathrm{O}_{15}$ & - \\
& $8 / 3$ & 4 & $\mathrm{SrBi}_{4} \mathrm{Ti}_{4} \mathrm{O}_{15}$ & 135 \\
& 3 & $15 / 4$ & $\mathrm{Bi}_{5} \mathrm{Ti}_{3} \mathrm{FeO}_{15}$ & 135
\end{tabular}

phase). Nonetheless, we can still consider $14 / \mathrm{mmm}$ as a hypothetical reference structure, which first-principles calculations have shown to be unstable to numerous structural distortions. ${ }^{20}$ The strongest instability is a polar $\mathrm{E}_{\mathrm{u}}\left(\Gamma_{5}{ }^{-}\right)$mode dominated by a displacements of $\mathrm{W}$ against the oxygen octahedra in the perovskite layers. Two other relevant instabilities are $\mathrm{X} \mathrm{X}_{2}{ }^{+}$mode related to rotations of oxygen octahedra around the $c$-axis and $\mathrm{a} \mathrm{X}_{3}{ }^{+}$mode related to tilts of oxygen octahedra around the $a$-axis. The intermediate $B 2 c b$ phase is reached from the combination of $\mathrm{\Gamma}_{5}{ }^{-}$and $\mathrm{X}_{3}{ }^{+}$motions, while the $P 2{ }_{1} a b$ phase requires the additional appearance of a $\mathrm{X}_{2}^{+}$distortion. Although the co-existence of these modes allows a priori for additional kinds of atomic displacements through different trilinear coupling terms, the structures of the ferroelectric phases are dominated by displacements arising from the initial instabilities $\left(\mathrm{\Gamma}_{5}{ }^{-}, \mathrm{X}_{3}{ }^{+}\right.$and $\left.\mathrm{X}_{2}{ }^{+}\right)$. Inspection of the energy landscape highlights that the bi-quadratic couplings between $\Gamma_{5}{ }^{-}, \mathrm{X}_{2}{ }^{+}$and $\mathrm{X}_{3}{ }^{+}$motions are positive but very weak so that these modes behave rather independently. The spontaneous polarization $P_{\mathrm{s}} \approx 48 \mu \mathrm{C} \mathrm{cm}^{-2}$ is mainly produced by the unstable $\Gamma_{5}{ }^{-}$mode and so arises from the motion of $\mathrm{W}$ and $\mathrm{O}$ in the perovskite block. An additional contribution could also come from a rigid motion of the $\mathrm{Bi}_{2} \mathrm{O}_{2}$ layers relative to the perovskite-like blocks, associated with a harder mode. ${ }^{127}$

$\mathrm{SrBi}_{2} \mathrm{Ta}_{2} \mathrm{O}_{9}(m=2, \mathrm{SBT})$ crystallizes at high temperature in the prototype $14 / \mathrm{mmm}$ phase. ${ }^{15,125}$ Similarly to BWO, this phase exhibits various structural instabilities ${ }^{136}$ including a polar $\mathrm{E}_{\mathrm{u}}$ mode associated with an anti phase displacement of the $\mathrm{Bi}$ atoms and the perovskite blocks (sometimes referred to as a rigid layer mode ${ }^{127,137}$ ) and $\mathrm{a}_{3}{ }^{-}$mode associated with tilts of the oxygen octahedra along the $a$-axis. In SBT, the strongest instability is the $\mathrm{X}_{3}{ }^{-}$mode and its condensation brings the system to an intermediate non-polar Amam phase. The $\mathrm{X}_{3}{ }^{-}$ and $\mathrm{E}_{\mathrm{u}}$ modes have a strong positive biquadratic coupling the condensation of both modes simultaneously is energetically unfavored - which suggests that Amam should be the ground-state. It was shown however that a strong trilinear coupling between $\mathrm{X}_{3}{ }^{-}, \mathrm{E}_{\mathrm{u}}$ and a hard $\mathrm{X}_{2}{ }^{+}$mode, involving only oxygen motion within the $\left[\mathrm{Bi}_{2} \mathrm{O}_{2}\right]$ layer, drives the system into a ground-state ferroelectric structure $A 2_{1} \mathrm{am}$ combining all three modes. $\mathrm{SrBi}_{2} \mathrm{Nb}_{2} \mathrm{O}_{9}$, isomorphous to $\mathrm{SBT}$, has the same ground-state space group but does not go through an intermediate phase: it displays a direct phase transition from I4/ 
$\mathrm{mmm}$ to $A 2_{1} \mathrm{am} \cdot{ }^{17,138}$ Intermediate phases have also not been detected in $\mathrm{Bi}_{5} \mathrm{Ti}_{3} \mathrm{FeO}_{15}$ or $\mathrm{Bi}_{4} \mathrm{Ti}_{3} \mathrm{O}_{12}$, both of which seem to exhibit a direct phase transition from the high-temperature tetragonal to a ferroelectric phase. Similarly, an Amam intermediate phase has been detected experimentally in $\mathrm{SrBi}_{4} \mathrm{Ti}_{4} \mathrm{O}_{15}$ in the region $550-650{ }^{\circ} \mathrm{C}^{135}$ but not in its analogue $\mathrm{Bi}_{5} \mathrm{Ti}_{3} \mathrm{FeO}_{15}{ }^{61}$

$\mathrm{Bi}_{4} \mathrm{Ti}_{3} \mathrm{O}_{12}(m=3)$ was originally suggested to exhibit a single phase transition from the $14 / \mathrm{mmm}$ high temperature phase to a $B 1 a 1$ ferroelectric monoclinic ground-state. ${ }^{123}$ However this study lacked the small temperature intervals necessary to detect the possible intermediate phases. Indeed, two more recent studies have suggested differing phase transition sequences ${ }^{139,140}$ and the mechanism of the phase transition is not yet fully understood. Theoretical studies suggest that it may involve a complex interplay of six different normal modes, belonging to four different irreducible representations. The primary instabilities consist of a polar $\mathrm{E}_{\mathrm{u}}$ mode located in the center of the perovskite block and dominated by $\mathrm{Bi}$ and $\mathrm{O}$ motions, $\mathrm{a} \mathrm{X}_{3}{ }^{+}$mode related to tilts of the oxygen octahedra along $a$ and $\mathrm{a}_{2}^{+}$mode associated with oxygen rotations around $c$. These three modes have the correct symmetry properties to lower the symmetry from $14 / \mathrm{mmm}$ to $B 1 \mathrm{a} 1$. Harder modes nevertheless do appear in the ground-state. The assumption of simultaneous condensation of the different order parameters through the so-called avalanche transition was made, but first-principles energy and phonon calculations of this compound failed to show any features that would favor such a mechanism. ${ }^{141}$ The true nature of any intermediate phases in $\mathrm{Bi}_{4} \mathrm{Ti}_{3} \mathrm{O}_{12}$ is thus yet to be established.

\subsection{Dion-Jacobson phases}

The crystal chemistry of the Dion-Jacobson phases has not been as extensively investigated as the Ruddlesden-Popper and Aurivillius phases. However, inspired by earlier experimental work, there has been a recent resurgence of interest (both from theorists and experimentalists) in Dion-Jacobson materials. Similar to Ruddlesden-Popper phases, the DionJacobson phases also form a homologous series with general formula $\mathrm{A}^{\prime}\left[\mathrm{A}_{n-1} \mathrm{~B}_{n} \mathrm{O}_{3 n+1}\right]$ for $n>2$, whereas the $n=1$ member has the formula $\mathrm{ABO}_{4}$ (most known $n=1$ materials are fluorides, however ${ }^{142}$ ). The $\mathrm{A}^{\prime}$ cation separates the perovskite-like blocks in Dion-Jacobson phases with $n>1$ and is typically an alkali cation, but can also be a transition metal halide complex, such as $(\mathrm{MnCl})^{+} \cdot{ }^{143-146}$ Among the oxide $n>1$ DionJacobson phases we focus on below, compositions with $\mathrm{A}=\mathrm{a}$ rare earth or $\mathrm{Bi}^{3+}$ and $\mathrm{B}=\mathrm{Nb}$ or Ta have been synthesized (oxyfluoride Dion-Jacobson phases are also known ${ }^{147-149}$ ).

As with the Aurivillius phases above, the lattice dynamics of the Dion-Jacobson phases cannot be linked to that of a parent perovskite phase because the material that makes up the perovskite-like blocks as $n \rightarrow \infty$ in the Dion-Jacobson phases does not exist independently as a bulk perovskite. For example, for a Dion-Jacobson phase with formula $\mathrm{Cs}\left[\mathrm{La}_{n-1}\right.$ $\left.\mathrm{Nb}_{n} \mathrm{O}_{3 n+1}\right]$ the perovskite-like blocks are composed of $\mathrm{La}-\mathrm{O}$ and $\mathrm{Nb}-\mathrm{O}$ layers, but there is no perovskite with formula
$\mathrm{LaNbO}_{3}$. A composition with $\mathrm{LaNbO}_{4}$ would be allowed and indeed this is the hypothetical $n=1$ member of the series; this stoichiometry is known to adopt a different structure. Hence, there does not seem to be any simple choice of fundamental building block for the Dion-Jacobson phases in the same sense that there is for the A-site ordered double perovskites and Ruddlesden-Popper phases. However, we can still obtain information about trends by comparing the behavior of different members of the family and this is the approach we take here.

The undistorted prototype structure for the Dion-Jacobson phases varies according to the identity of the $\mathrm{A}^{\prime}$ cation. For smaller $\mathrm{A}^{\prime}$ cations ( $\mathrm{Li}, \mathrm{Na}$ ), the perovskite blocks in adjacent layers are offset from each other by $1 / 2[110]$ and the undistorted prototype has $14 / \mathrm{mmm}$ symmetry, like the RuddlesdenPopper phases. The prototype has $\mathrm{Cmcm}$ symmetry for slightly larger cations (such as $\mathrm{K}$ ), in which case the perovskite blocks in adjacent layers are offset by $1 / 2[100]$ (or $1 / 2[010]$ ). All of the materials discussed below contain $\mathrm{Cs}$ or $\mathrm{Rb}$ at the $\mathrm{A}^{\prime}$ site and here the prototype has $P 4 / \mathrm{mmm}$ symmetry with the perovskite blocks in adjacent layers directly aligned.

$\mathrm{CsBiNb}_{2} \mathrm{O}_{7}$ and $\mathrm{RbBiNb}_{2} \mathrm{O}_{7}$ are perhaps the most wellstudied polar Dion-Jacobson materials. They were first synthesized relatively recently by Subramanian and co-workers ${ }^{150}$ and characterized as orthorhombic with $a_{\mathrm{O}} \sim b_{\mathrm{O}} \sim 2 a_{\mathrm{T}}$ and $c_{\mathrm{O}}$ $\sim 2 c_{\mathrm{T}}$, where $a_{\mathrm{O}}, b_{\mathrm{O}}$ and $c_{\mathrm{O}}$ refer to the lattice parameters of the observed orthorhombic structure and $a_{\mathrm{T}}$ and $c_{\mathrm{T}}$ refer to the lattice parameters of the undistorted $P 4 / \mathrm{mmm}$ prototype. Although $\mathrm{CsBiNb}_{2} \mathrm{O}_{7}$ and $\mathrm{RbBiNb}_{2} \mathrm{O}_{7}$ were identified as having a larger unit cell and lower symmetry than the prototype phase, neither of the materials was initially reported as polar. The subsequent powder neutron diffraction study of Snedden et $a l^{31}$ showed that $\mathrm{CsBiNb}_{2} \mathrm{O}_{7}$ is indeed polar (along with $\mathrm{CsNdNb}_{2} \mathrm{O}_{7}$ ) with space group $P m c 2_{1}$ and lattice parameters $a_{\mathrm{O}}$ $\sim b_{\mathrm{O}} \sim \sqrt{2} a_{\mathrm{T}}$ and $c_{\mathrm{O}} \sim c_{\mathrm{T}}$, i.e., the $a$ and $b$ lattice parameters are enlarged compared to the undistorted phase but in a different sense to that reported by Subramanian. In their analysis of the structures of $\mathrm{CsBiNb}_{2} \mathrm{O}_{7}$ and $\mathrm{CsNdNb}_{2} \mathrm{O}_{7}$ Snedden noted the "large polar displacements of the perovskite A cation with cooperative octahedral tilting" corresponding to the $a^{-} a^{-} c^{+}$Glazer tilt pattern. The first-principles calculations of Fennie and Rabe ${ }^{151}$ confirmed the importance of octahedral rotations in giving rise to the polar structure, but the mechanism through which the polar phase emerges from the undistorted $P 4 / \mathrm{mmm}$ prototype was not elucidated. In addition, although experiments had confirmed that both $\mathrm{CsBiNb}_{2} \mathrm{O}_{7}$ and $\mathrm{CsNdNb}_{2} \mathrm{O}_{7}$ belong to polar space groups, neither material appeared to be ferroelectric (polarization switching was not demonstrated $^{31,32}$ ). Goff ${ }^{32}$ reported a structural study of $\mathrm{CsBiNb}_{2} \mathrm{O}_{7}$ by powder neutron diffraction up to $900{ }^{\circ} \mathrm{C}$, but saw no significant change in the orthorhombic or polar distortion; the recent study of dielectric properties by Chen et al. ${ }^{34}$ suggests a $T_{\mathrm{C}}$ of $1033{ }^{\circ} \mathrm{C}$, so a further diffraction-based study is now prompted in order to elucidate the details of this transition.

The polarity of both $\mathrm{CsBiNb}_{2} \mathrm{O}_{7}$ and $\mathrm{RbBiNb}_{2} \mathrm{O}_{7}$ is most often ascribed to the stereoactive $\mathrm{Bi}^{3+}$ lone pair. However, this 
cannot explain why $\mathrm{CsNdNb}_{2} \mathrm{O}_{7}$ adopts a polar structure since this material does not contain any lone pair cations. It is also somewhat curious that of the many known $n=2$ Dion-Jacobson phases, only a few have been definitively identified as belonging to polar space groups. A recent first-principles study $^{35}$ of a series of $n=2$ Dion-Jacobson phases $\left(\mathrm{A}^{\prime}=\mathrm{Rb}, \mathrm{Cs}\right.$, $\mathrm{A}=\mathrm{La}, \mathrm{Nd}, \mathrm{Y}, \mathrm{Bi}, \mathrm{B}=\mathrm{Nb}$ ) predicted that all of these materials should adopt the same polar $P m c 2_{1}$ space group as the Bi-containing compounds, even $\mathrm{CsLaNb}_{2} \mathrm{O}_{7}$, which has only ever been characterized in the undistorted $P 4 / \mathrm{mmm}$ phase and is often considered the archetype of this structure. Ref. 35 also showed that the transition from $P 4 / \mathrm{mmm}$ to $P m c 2_{1}$ is driven by the same trilinear coupling mechanism found in the materials families discussed above: two octahedral rotation distortions, one similar to the $a^{0} a^{0} c^{+}$Glazer tilt pattern (transforming like the irrep $\mathrm{M}_{2}^{+}$) and the other to $a^{-} a^{-} c^{0}\left(\mathrm{M}_{5}{ }^{-}\right)$, couple to a polar mode $\left(\Gamma_{5}{ }^{-}\right)$to give rise to a macroscopic polarization. As with the other layered materials discussed in this Perspective, the octahedral rotations optimize the A-site coordination environment, which is under-bonded in $P 4 / \mathrm{mmm}$. This is the case even in the Bi-containing compounds, i.e., although the lone pair on Bi enhances the magnitude of the polarization in the polar phase, these Dion-Jacobson materials can undergo polar distortions even in the absence of stereoactive cations.

Fig. 6 shows the layer-resolved polarization for $P m c 2_{1}$ $\mathrm{CsLaNb}_{2} \mathrm{O}_{7}$ from first-principles calculations. ${ }^{35}$ In contrast to the pattern of anti-polar displacements found in the double perovskites and Ruddlesden-Popper phases, here the layer polarizations are all aligned and hence the macroscopic polarization does not arise from an incomplete cancelation of oppositely oriented A-O layer polarizations. The reason for this is not difficult to understand: there is only one A-O layer in the $n=2$ Dion-Jacobson phases, as opposed to at least two in the double perovskites and Ruddlesden-Popper phases. Hence, even though A-site under bonding is ultimately responsible for driving the transition from $P 4 / \mathrm{mmm}$ to $P m c 2_{1}$, the main contribution to the polarization actually comes from the B-O layers, simply because there are two B-O layers per unit cell. In
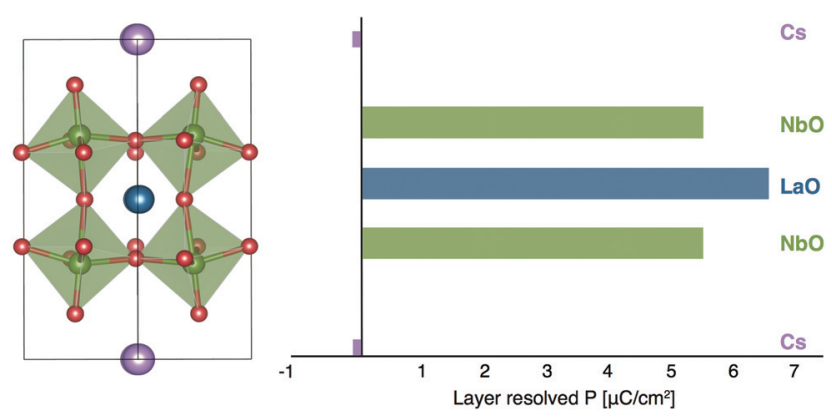

Fig. 6 Layer-resolved polarization of $\mathrm{Pmc}_{1} \mathrm{CsLaNb}_{2} \mathrm{O}_{7}$ from first-principles calculations. ${ }^{35}$ Note that even though the main contribution to the polarization comes from the $\mathrm{Nb}-\mathrm{O}$ layers, the ' $\mathrm{d}^{0}$-ness' of $\mathrm{Nb}$ plays no part in driving the transition from the undistorted $P 4 / \mathrm{mmm}$ phase to $P_{m c 2}$. See ref. 35 for further details. Reprinted with permission from Inorganic Chemistry, 2014, 53, 3769-3777. Copyright 2014 American Chemical Society. addition, similar to the Ruddlesden-Popper phases, only even$n$ Dion-Jacobson phases will be polar, i.e., the $a^{-} a^{-} c^{+}$tilt pattern does not give rise to a polar space group in odd- $n$ Dion-Jacobson phases, ${ }^{152,153}$ but a polar structure may emerge through some other mechanism.

The recent flurry of results and interest in Dion-Jacobson phases has reinvigorated experimental efforts. Polarization switching has now been demonstrated in both $\mathrm{CsBiNb}_{2} \mathrm{O}_{7}{ }^{34}$ and $\mathrm{RbBiNb}_{2} \mathrm{O}_{7}{ }^{33,34}$ and the experimentally observed polarizations are generally in good agreement with those predicted from theory. Several Dion-Jacobson materials that had previously only been reported in the undistorted $P 4 / \mathrm{mmm}$ structure have been re-examined and preliminary results suggest that the list of polar Dion-Jacobson phases may be growing. However, the nature of the high-temperature structural behaviour of the Dion-Jacobson family is also much less well studied than that of the Ruddlesden-Popper and Aurivillius phases. Apart from the study of $\mathrm{CsBiNb}_{2} \mathrm{O}_{7}$ by Goff et al. ${ }^{32}$ which unfortunately did not reach $T_{\mathrm{C}}$, we are only aware of one other variable temperature crystallographic study: Geselbracht et $a l .{ }^{154}$ reported from powder XRD that $\mathrm{RbCa}_{2} \mathrm{Nb}_{3} \mathrm{O}_{10}$ retains tetragonal symmetry throughout the range $\mathrm{RT}<T<1000{ }^{\circ} \mathrm{C}$. In addition, the presence of alkali metals in the inter-layer regions makes these materials intrinsically susceptible to ionexchange and water-uptake phenomena, thus exacerbating the difficulties in obtaining robust ferroelectric behavior. Further studies might therefore target compositions with, for example, $\mathrm{Ba}^{2+}$ in the interlayer $\mathrm{A}^{\prime}$ sites. Examples of such materials are known, such as $\mathrm{BaSrTa}_{2} \mathrm{O}_{7}$; previous studies ${ }^{155}$ have suggested non-polar symmetry (Immm) at ambient temperature.

\section{Summary and outlook}

Hybrid improper ferroelectricity and the trilinear coupling mechanism are much more than a simple academic curiosity. Rather, they provide a unifying framework for understanding the crystal chemistry of polar structures in many families of layered perovskites and a powerful pathway to engineer new or enhanced functional properties. Independent of the primary order parameters and the improper nature of the phase transition, the trilinear coupling of lattice modes in the low-symmetry phase appears in itself as a key feature to be exploited. As anticipated by Bousquet et al., ${ }^{47}$ the linear coupling between polar and non-polar distortions offers a unique opportunity to tune with an electric field non-polar distortions and the properties linked to them. A concrete realization of this is, for example, the predicted electric field-controllable magnetization in Ruddlesden-Popper $\mathrm{Ca}_{3} \mathrm{Mn}_{2} \mathrm{O}_{7} \cdot{ }^{50}$

From the experimental side, the present overview shows that a combination of first-principles calculations, guided by symmetry analysis of all possible distortion mode pathways provides an essential guide for the experimental inorganic chemist in targeting suitable compositions likely to display ferroelectricity in the various families of layered perovskites. Crystallographic studies must become more detailed and 
reliable, both in characterizing the ambient temperature phases and the nature of the high-temperature phase transitions towards and into the paraelectric state. Such studies require either single crystal X-ray or the highest quality powder neutron diffraction data. In addition, care must be taken in processing of ceramics suitable for robust and reliable dielectric data to be obtained.

Most efforts so far have focused on specific trilinear terms involving the polarization and antiferrodistortive oxygen motions linked to Glazer's tilt patterns $a^{0} a^{0} c^{-}$and $a^{0} a^{0} c^{+}$. Trilinear terms can also couple the polarization to other types of atomic motions like Jahn-Teller ${ }^{156-158}$ or anti-polar ${ }^{159}$ distortions. In the highly strained $P m c 2_{1}$ phase of $\mathrm{BiFeO}_{3}$ for instance, ${ }^{159}$ a trilinear term involving the polarization, oxygen rotations and anti-polar distortion was predicted to allow for electric control of the magnetization. In $\mathrm{AVO}_{3} / \mathrm{A}^{\prime} \mathrm{VO}_{3} 1 / 1$ superlattices, ${ }^{160}$ a term linking two Jahn-Teller distortions to the polarization was recently shown to be able to produce an outof-plane polarization and to allow for electric-field induced magnetic phase transitions. Layered perovskites allow for various trilinear mechanisms and offer a still widely unexplored playground for the coupling of functional properties and the appearance of unexpected phenomena.

\section{Acknowledgements}

We thank current and past students, postdocs and collaborators for insightful conversations. N. A. B. was supported by The Welch Foundation under Grant. no. F-1803. J. M. R. acknowledges support from the Penn State Center for Nanoscience, National Science Foundation grant no. DMR-1420620. Ph.G. acknowledges a research Professorship of the Francqui Foundation and financial supports of the ARC project TheMoTherm and FNRS project HiT4FiT.

\section{References}

1 M. E. Lines and A. M. Glass, Principles and Applications of Ferroelectrics and Related Materials, Oxford University Press, 1977.

2 P. S. Halasyamani and K. R. Poeppelmeier, Chem. Mater., 1998, 10, 2753-2769.

3 Physics of Ferroelectrics A Modern Perspective, ed. K. M. Rabe, C. H. Ahn and J. Triscone, Springer, 2007.

4 J. Wang, J. B. Neaton, H. Zheng, V. Nagarajan, S. B. Ogale, B. Liu, D. Viehland, V. Vaithyanathan, D. G. Schlom, U. V. Waghmare, N. A. Spaldin, K. M. Rabe, M. Wuttig and R. Ramesh, Science, 2003, 299, 1719-1722.

5 W. Eerenstein, N. D. Mathur and J. F. Scott, Nature, 2006, 442, 759-765.

6 I. B. Bersuker, Phys. Lett., 1966, 20, 589-590.

7 I. B. Bersuker and B. G. Vekhter, Ferroelectrics, 1978, 19, 137-150.

8 J. K. Burdett, Inorg. Chem., 1981, 20, 1959-1962.
9 R. E. Cohen, Nature, 1992, 358, 136-138.

10 P. Ghosez, X. Gonze and J.-P. Michenaud, Europhys. Lett., 1996, 33, 713.

11 D. J. Payne, R. G. Egdell, A. Walsh, G. W. Watson, J. Guo, P.-A. Glans, T. Learmonth and K. E. Smith, Phys. Rev. Lett., 2006, 96, 157403.

12 U. V. Waghmare, N. A. Spaldin, H. C. Kandpal and R. Seshadri, Phys. Rev. B: Condens. Matter, 2003, 67, 125111.

13 N. A. Benedek and C. J. Fennie, J. Phys. Chem. C, 2013, 117, 13339-13349.

14 B. Aurivillius, Ark. Kemi., 1950, 1, 463-480.

15 C. H. Hervoches, J. T. S. Irvine and P. Lightfoot, Phys. Rev. B: Condens. Matter, 2001, 64, 100102(R).

16 J. M. Perez-Mato, M. Aroyo, A. García, P. Blaha, K. Schwarz, J. Schweifer and K. Parlinski, Phys. Rev. B: Condens. Matter, 2004, 70, 214111.

17 P. Boullay, J. Tellier, D. Mercurio, M. Manier, F. J. Zuñiga and J. M. Perez-Mato, Solid State Sci., 2012, 14, 13671371.

18 C. A-Paz de Araujo, J. D. Cuchiaro, L. D. McMillan, M. C. Scott and J. F. Scott, Nature, 1995, 374, 627629.

19 J. F. Scott, Phys. World, 1995, 8, 45-50.

20 H. Djani, E. Bousquet, A. Kellou and P. Ghosez, Phys. Rev. B: Condens. Matter, 2012, 86, 054107.

21 H. Djani, P. Hermet and P. Ghosez, J. Phys. Chem. C, 2014, 118, 13514-13524.

22 R. L. Withers, J. G. Thompson and A. D. Rae, J. Solid State Chem., 1991, 94, 404-417.

23 S. N. Ruddlesden and P. Popper, Acta Crystallogr., 1957, 10, 538-539.

24 S. N. Ruddlesden and P. Popper, Acta Crystallogr., 1958, 11, 54-55.

25 M. M. Elcombe, E. H. Kisi, K. D. Hawkins, T. J. White, P. Goodman and S. Matheson, Acta Crystallogr., Sect. B: Struct. Sci., 1991, 47, 305-314.

26 N. Guiblin, D. Grebille, H. Leligny and C. Martin, Acta Crystallogr., Sect. C: Cryst. Struct. Commun., 2002, 58, i3-i5.

27 M. I. Lobanov, M. Greenblatt, E. N. Caspi, J. D. Jorgensen, D. V. Sheptyakov, B. H. Toby, C. E. Botez and P. W. Stephens, J. Phys.: Condens. Matter, 2004, 16, 53395348.

28 Y. Yoshida, S. Ikeda, H. Matsuhata, N. Shirakawa, C. H. Lee and S. Katano, Phys. Rev. B: Condens. Matter, 2005, 72, 054412.

29 M. Dion, M. Ganne and M. Tournoux, Mater. Res. Bull., 1981, 16, 1429-1435.

30 A. J. Jacobson, J. W. Johnson and J. T. Lewandowski, Inorg. Chem., 1985, 24, 3727-3729.

31 A. Snedden, K. S. Knight and P. Lightfoot, J. Solid State Chem., 2003, 173, 309-313.

32 R. J. Goff, D. Keeble, P. A. Thomas, C. Ritter, F. D. Morrison and P. Lightfoot, Chem. Mater., 2009, 21, 1296-1302. 
33 B. Li, M. Osada, T. C. Ozawa and T. Sasaki, Chem. Mater., 2012, 24, 3111-3113.

34 C. Chen, H. Ning, S. Lepadatu, M. Cain, H. Yan and M. J. Reece, J. Mater. Chem. C, 2015, 3, 19-22.

35 N. A. Benedek, Inorg. Chem., 2014, 53, 3769-3777.

36 M. C. Knapp and P. M. Woodward, J. Solid State Chem., 2006, 179, 1076-1085.

37 G. King and P. M. Woodward, J. Mater. Chem., 2010, 20, 5785-5796.

38 G. King, S. Thimmaiah, A. Dwivedi and P. M. Woodward, Chem. Mater., 2007, 19, 6451-6458.

39 J. M. Rondinelli and C. J. Fennie, Adv. Mater., 2012, 24, 1961-1968.

40 A. T. Mulder, N. A. Benedek, J. M. Rondinelli and C. J. Fennie, Adv. Funct. Mater., 2013, 23, 4810-4820.

41 J. Young and J. M. Rondinelli, Chem. Mater., 2013, 25, 4545-4550.

42 J. B. Neaton and K. M. Rabe, Appl. Phys. Lett., 2003, 82, 1586-1588.

43 M. Dawber, C. Lichtensteiger, M. Cantoni, M. Veithen, P. Ghosez, K. Jonhston, K. M. Rabe and J. Triscone, Phys. Rev. Lett., 2005, 95, 177601.

44 M. Dawber, N. Stucki, C. Lichtensteiger, S. Gariglio, P. Ghosez and J.-M. Triscone, Adv. Mater., 2007, 4153, 49.

45 M. Sepliarsky, S. R. Phillpot, D. Wolf, M. G. Stachiotti and R. L. Migoni, Phys. Rev. B: Condens. Matter, 2001, 64, R060101.

46 M. Sepliarsky, S. R. Phillpot, D. Wolf, M. G. Stachiotti and R. L. Migoni, J. Appl. Phys., 2001, 90, 4509.

47 E. Bousquet, M. Dawber, N. Stucki, C. Lichtensteiger, P. Hermet, S. Gariglio, J.-M. Triscone and P. Ghosez, Nature, 2008, 452, 732-736.

48 F. Lichtenberg, A. Herrnberger and K. Wiedenmann, Prog. Solid State Chem., 2008, 36, 253-387.

49 I. Etxebarria, J. M. Perez-Mato and P. Boullay, Ferroelectrics, 2010, 401, 17-23.

50 N. A. Benedek and C. J. Fennie, Phys. Rev. Lett., 2011, 106, 107204.

51 T. Fukushima, A. Stroppa, S. Picozzi and J. M. Perez-Mato, Phys. Chem. Chem. Phys., 2011, 13, 12186-12190.

52 M. Stengel, C. J. Fennie and P. Ghosez, Phys. Rev. B: Condens. Matter, 2012, 86, 094112.

53 H. J. Zhao, J. Íñiguez, W. Ren, X. M. Chen and L. Bellaiche, Phys. Rev. B: Condens. Matter, 2014, 89, 174101.

54 W. Cochran, Phys. Rev. Lett., 1959, 3, 412-414.

55 A. P. Levanyuk and D. G. Sannikov, Usp. Fiz. Nauk, 1974, 112, 561-589.

56 B. B. Van Aken, T. T. M. Palstra, A. Filippeti and N. A. Spaldin, Nat. Mater., 2004, 3, 164-170.

57 C. J. Fennie and K. M. Rabe, Phys. Rev. B: Condens. Matter, 2005, 72, 100103(R).

58 J. Varignon and P. Ghosez, Phys. Rev. B: Condens. Matter, 2013, 87, 140403.

59 P. Tolédano and J. Tolédano, Phys. Rev. B: Condens. Matter, 1976, 14, 3097-3109.
60 M. Stengel, N. A. Spaldin and D. Vanderbilt, Nat. Phys., 2009, 5, 304-308.

61 A. Snedden, C. H. Hervoches and P. Lightfoot, Phys. Rev. B: Condens. Matter, 2003, 67, 092102.

62 M. Dawber, K. M. Rabe and J. F. Scott, Rev. Mod. Phys., 2005, 77, 1083-1130.

63 I. Ponomareva, I. Naumov, I. Kornev, H. Fu and L. Bellaiche, Curr. Opin. Solid State Mater. Sci., 2005, 9, 114-121.

64 K. M. Rabe, Curr. Opin. Solid State Mater. Sci., 2005, 9, 122.

65 W. Duan and Z. R. Liu, Curr. Opin. Solid State Mater. Sci., 2006, 10, 40-51.

66 J. Junquera and P. Ghosez, J. Comput. Theor. Nanosci., 2008, 5, 2071.

67 J. Junquera and P. Ghosez, Nature, 2003, 422, 506-509.

68 M. Stengel and N. A. Spaldin, Nature, 2006, 443, 679-682.

69 I. Ponomareva, I. I. Naumov, I. Kornev, H. Fu and L. Bellaiche, Phys. Rev. B: Condens. Matter, 2005, 72, 140102(R).

70 M. Stengel, D. Vanderbilt and N. A. Spaldin, Nat. Mater., 2009, 8, 392.

71 C. Lichtensteiger, S. Fernandez-Pena, C. Weymann, P. Zubko and J.-M. Triscone, Nano Lett., 2014, 14, 42054211.

72 N. A. Pertsev, A. G. Zembilgotov and A. K. Tagantsev, Phys. Rev. Lett., 1998, 80, 1988-1991.

73 N. A. Pertsev, A. K. Tagantsev and N. Setter, Phys. Rev. B: Condens. Matter, 2000, 61, R825-R829.

74 K. J. Choi, M. Biegaslki, Y. L. Li, A. Sharan, J. Schubert, R. Uecker, P. Reiche, Y. B. Chen, X. Q. Pan, V. Gopalan, L. Q. Chen, D. G. Schlom and C. B. Eom, Science, 2004, 306, 1005-1009.

75 J. H. Haeni, P. Irvin, W. Chang, R. Uecker, P. Reiche, Y. L. Li, S. Choudhury, W. Tian, M. E. Hawley, B. Craigo, A. K. Tagantsev, X. Q. Pan, S. K. Streiffer, L. Q. Chen, S. W. Kirchoefer, J. Levy and D. G. Schlom, Nature, 2004, 430, 758-761.

76 O. Diéguez, K. M. Rabe and D. Vanderbilt, Phys. Rev. B: Condens. Matter, 2005, 72, 144101.

77 P. Aguado-Puente and J. Junquera, Phys. Rev. B: Condens. Matter, 2012, 85, 184105.

78 P. Zubko, N. Jecklin, A. Torres-Pardo, P. Aguado-Puente, A. Gloter, C. Lichtensteiger, J. Junquera, O. Stéphan and J.-M. Triscone, Nano Lett., 2012, 12, 2846-2851.

79 N. Sai, B. Meyer and D. Vanderbilt, Phys. Rev. Lett., 2000, 84, 5636-5639.

80 H. N. Lee, H. M. Christen, M. F. Chisholm, C. M. Rouleau and D. H. Lowndes, Nature, 2005, 433, 395-399.

81 S. M. Nakhmanson, K. M. Rabe and D. Vanderbilt, Appl. Phys. Lett., 2005, 87, 102906.

82 C. Lichtensteiger, P. Zubko, M. Stengel, P. Aguado-Puente, J. M. Triscone, P. Ghosez and J. Junquera, in Oxides Ultrathin Films: Science and Technology, ed. G. Pacchioni and S. Valeri, Wiley-VCH, Weinheim, Germany, 2011, pp. 265-308. 
83 P. Aguado-Puente, P. Garcia-Fernandez and J. Junquera, Phys. Rev. Lett., 2011, 107, 217601.

84 J. Young and J. M. Rondinelli, Chem. Mater., 2013, 25, 4545-4550.

85 G. Gou and J. M. Rondinelli, Adv. Mater. Interfaces, 2014, 1, 1400042 .

86 H. Sim, S. Cheong and B. Kim, Phys. Rev. B: Condens. Matter, 2013, 88, 014101.

87 L. Bellaiche and J. Íñiguez, Phys. Rev. B: Condens. Matter, 2013, 88, 014104.

88 X. Lu, X. Gong and H. Xiang, Comput. Mater. Sci., 2014, 91, 310-314.

89 Z. Zanolli, J. Wojdeł, J. Íñiguez and P. Ghosez, Phys. Rev. B: Condens. Matter, 2013, 88, 060102.

90 O. Diéguez, O. González-Vázquez, J. Wojdeł and J. Íñiguez, Phys. Rev. B: Condens. Matter, 2011, 83, 094105.

91 E. Bousquet and N. A. Spaldin, Phys. Rev. Lett., 2011, 107, 197603.

92 S. Ghosh, H. Das and C. J. Fennie, ArXiv e-prints, 2014.

93 J. Alaria, P. Borisov, M. S. Dyer, T. D. Manning, S. Lepadatu, M. G. Cain, E. D. Mishina, N. E. Sherstyuk, N. A. Ilyin, J. Hadermann, D. Lederman, J. B. Claridge and M. J. Rosseinsky, Chem. Sci., 2014, 5, 1599-1610.

94 Y. F. Nie, Y. Zhu, C.-H. Lee, L. F. Kourkoutis, J. A. Mundy, J. Junquera, P. Ghosez, D. J. Baek, S. Sung, X. X. Xi, K. M. Shen, D. A. Muller and D. G. Schlom, Nat. Commun., 2014, 5, 4530 .

95 J. H. Lee, G. Luo, I. C. Tung, S. H. Chang, Z. Luo, M. Malshe, M. Gadre, A. Bhattacharya, S. M. Nakhmanson, J. A. Eastman, H. Hong, J. Jellinek, D. Morgan, D. D. Fong and J. W. Freeland, Nat. Mater., 2014, 13, 879-883.

96 N. G. Zamkova, V. S. Zhandun and V. I. Zinenko, Phys. Status Solidi B, 2013, 250, 1888-1897.

97 J. Young and J. M. Rondinelli, Phys. Rev. B: Condens. Matter, 2014, 89, 174110.

98 G. King, A. Wills and P. Woodward, Phys. Rev. B: Condens. Matter, 2009, 79, 224428.

99 C. De, T. H. Kim, K. H. Kim and A. Sundaresan, Phys. Chem. Chem. Phys., 2014, 16, 5407-5411.

100 H. J. Zhao, W. Ren, Y. Yang, J. I. Niguez, X. M. Chen and L. Bellaiche, Nat. Commun., 2014, 1, 4021.

101 H. J. Zhao, X. Q. Liu, X. M. Chen and L. Bellaiche, Phys. Rev. B: Condens. Matter, 2014, 90, 195147.

102 A. Tarancón, M. Burriel, J. Santiso, S. J. Skinner and J. A. Kilner, J. Mater. Chem., 2010, 20, 3799-3813.

103 A. Chroneos, B. Yildiz, A. Tarancón, D. Parfitt and J. A. Kilner, Energy Environ. Sci., 2011, 4, 2774-2789.

104 J. G. Bednorz and K. A. Muller, Z. Phys. B: Condens. Matter, 1986, 64, 189-193.

105 Y. Maeno, H. Hashimoto, K. Yoshida, S. Nishizaki, T. Fujita and J. G. Bednorz, Nature, 1994, 372, 532-534.

106 Y. S. Oh, X. Luo, F. Huang, Y. Wang and S. Cheong, Nat. Mater., 2015, DOI: 10.1038/nmat4168.

107 M. S. Senn, A. Bombardi, C. A. Murray, C. Vecchini, A. Scherillo, X. Luo and S. W. Cheong, Phys. Rev. Lett., 2015, 035701.
108 M. J. Pitcher, P. Mandal, M. S. Dyer, J. Alaria, P. Borisov, H. Niu, J. B. Claridge and M. J. Rosseinsky, Science, 2015, 347, 420-424.

109 N. A. Benedek, A. T. Mulder and C. J. Fennie, J. Solid State Chem., 2012, 195, 11-20.

110 P. V. Balachandran, D. Puggioni and J. M. Rondinelli, Inorg. Chem., 2014, 53, 336-348.

111 H. Akamatsu, K. Fujita, T. Kuge, A. Sen Gupta, A. Togo, S. Lei, F. Xue, G. Stone, J. M. Rondinelli, L.-Q. Chen, I. Tanaka, V. Gopalan and K. Tanaka, Phys. Rev. Lett., 2014, 112, 187602.

112 N. Sharma, B. Kennedy, M. Elcombe, Y. Liu and C. Ling, J. Phys.: Condens. Matter, 2008, 20, 025215.

113 B. Zulhadjri, B. Prijamboedi, A. Nugroho, et al., J. Solid State Chem., 2011, 184, 1318-1323.

114 F. Yang, P. Su, C. Wei, X. Chen, C. Yang and W. Cao, J. Appl. Phys., 2011, 110, 126102.

115 S. Tinte and M. Stachiotti, Phys. Rev. B: Condens. Matter, 2012, 85, 224112.

116 Y. Birenbaum and C. Ederer, Phys. Rev. B: Condens. Matter, 2014, 90, 214109.

117 J.-C. Champarnaud-Mesjard, B. Frit and A. Watanabe, J. Mater. Chem., 1999, 9, 1319-1322.

118 P. Millan, A. Ramirez and A. Castro, J. Mater. Sci. Lett., 1995, 14, 1657-1660.

119 Y. González-Abreu, A. Peláiz-Barranco, J. Guerra, Y. Gagou and P. Saint-Grégoire, J. Mater. Sci., 2014, 49, 7437-7444.

120 N. Kim, R.-N. Vannier and C. P. Grey, Chem. Mater., 2005, 17, 1952-1958.

121 A. Kudo and S. Hijii, Chem. Lett., 1999, 1999, 1103-1104.

122 K. Yee, T. A. Albright, D. Jung and M.-H. Whangbo, Angew. Chem., Int. Ed. Engl., 1989, 28, 750-751.

123 C. H. Hervoches and P. Lightfoot, Chem. Mater., 1999, 11, 3359.

124 J. F. Dorrian, R. E. Newnham and D. K. Smith, Ferroelectrics, 1971, 3, 17.

125 R. E. Newnham, R. Wolfe, R. Horsey, F. Diazcolon and M. Kay, Mater. Res. Bull., 1973, 8, 1183.

126 A. D. Rae, J. G. Thompson and R. L. Withers, Acta Crystallogr., Sect. B: Struct. Sci., 1991, 47, 870.

127 R. Machado, M. G. Stachiotti and A. H. Tera, Phys. Rev. B: Condens. Matter, 2004, 70, 214112.

128 N. A. McDowell, K. S. Knight and P. Lightfoot, Chem. Eur. J., 2006, 12, 1493.

129 K. Muramatsu, M. Shimazu, J. Tanaka and S. Horiuchi, J. Solid State Chem., 1981, 36, 179-182.

130 D. Mercurio, G. Trolliard, T. Hansen and J. Mercurio, Int. J. Inorg. Mater., 2000, 2, 397-406.

131 L. Shebanov, V. Osipyan and E. Freidenfield, Izv. Akad. Nauk. Arm. SSSR, Neorg. Mater., 1982, 18, 305.

132 V. K. Yanovskii and V. I. Voronkova, Phys. Status Solidi A, 1986, 93, 57-66.

133 H. Yan, H. Zhang, R. Ubic, M. Reece, J. Liu, Z. Shen and Z. Zhang, Adv. Mater., 2005, 17, 1261-1265.

134 A. Ramaman, J. G. Bishnan, M. Uppal, D. Jefferson and C. Rao, Proc. R. Soc. London, Ser. A, 1984, 395, 127-139. 
135 C. H. Hervoches, A. Snedden, R. Riggs, S. H. Kilcoyne, P. Manuel and P. Lightfoot, J. Solid State Chem., 2002, 164, 280.

136 J. M. Perez-Mato, M. Aroyo, A. García, P. Blaha, K. Schwarz, J. Schweifer and K. Parlinski, Phys. Rev. B: Condens. Matter, 2004, 70, 214111.

137 M. G. Stachiotti, C. O. Rodriguez, C. Ambrosch-Draxl and N. E. Christensen, Phys. Rev. B: Condens. Matter, 2000, 61, 14434-14439.

138 K. Miura, J. Korean Phys. Soc., 2003, 42, S1244S1247.

139 Q. Zhou, B. J. Kennedy and C. J. Howard, Chem. Mater., 2003, 15, 5025-5028.

140 M. Iwata, K. Ando, M. Maeda and Y. Ishibashi, J. Phys. Soc. Jpn., 2013, 82, 025001.

141 J. M. Perez-Mato, P. Blaha, K. Schwarz, M. Aroyo, D. Orobengoa, I. Etxebarria and A. García, Phys. Rev. B: Condens. Matter, 2008, 77, 184104.

142 M. Leblanc, V. Maisonneuve and A. Tressaud, Chem. Rev., 2014, 115, 1191-1254.

143 T. A. Kodenkandath, J. N. Lalena, W. L. Zhou, E. E. Carpenter, C. Sangregorio, A. U. Falster, W. B. Simmons, C. J. O'Connor and J. B. Wiley, J. Am. Chem. Soc., 1999, 121, 10743-10746.

144 T. A. Kodenkandath, A. S. Kumbhar, W. L. Zhou and J. B. Wiley, Inorg. Chem., 2001, 40, 710-714.

145 L. Viciu, G. Caruntu, N. Royant, J. Koenig, W. L. Zhou, T. A. Kodenkandath and J. B. Wiley, Inorg. Chem., 2002, 41, 3385-3388.
146 L. Viciu, V. O. Golub and J. B. Wiley, J. Solid State Chem., 2003, 175, 88-93.

147 J. Choy, J. Kim, S. Kim and J. Sohn, Chem. Mater., 2001, 13, 906-912.

148 G. Caruntu, L. Spinu and J. B. Wiley, Mater. Res. Bull., 2002, 37, 133-140.

149 Y. Kobayashi, M. Tian, M. Eguchi and T. E. Mallouk, J. Am. Chem. Soc., 2009, 131, 9849-9855.

150 M. A. Subramanian, J. Gopalakrishnan and A. W. Sleight, Mater. Res. Bull., 1988, 23, 837-842.

151 C. J. Fennie and K. M. Rabe, Appl. Phys. Lett., 2006, 88, 262902.

152 K. S. Aleksandrov, Crystallogr. Rep., 1995, 40, 251-272.

153 K. S. Aleksandrov and J. Bartolomé, Phase Transitions, 2001, 74, 255-335.

154 M. J. Geselbracht, R. I. Walton, E. S. Cowell, F. Millange and D. O'Hare, Chem. Mater., 2002, 14, 4343-4349.

155 F. Le Berre, M. P. Crosnier-Lopez and J. L. Fourquet, Solid State Sci., 2004, 6, 53-59.

156 A. Stroppa, P. Barone, P. Jain, J. M. Perez-Mato and S. Picozzi, Adv. Mater., 2013, 25, 2284.

157 Y. Tian, A. Stroppa, Y.-S. Chai, P. Barone, M. Perez-Mato, S. Picozzi and Y. Sun, Phys. Status Solidi RRL, 2014, 1, 1-6.

158 N. C. Bristowe, J. Varignon, D. Fontaine, E. Bousquet and P. Ghosez, Nat. Commun., 2015, in press.

159 Y. Yang, J. Íñiguez, A.-J. Mao and L. Bellaiche, Phys. Rev. Lett., 2014, 112, 057202.

160 J. Varignon, N. C. Bristowe, E. Bousquet and P. Ghosez, 2014, arXiv:1409.8422. 